\title{
Topography of the N400: brain electrical activity reflecting semantic expectancy *
}

\author{
Tim Curran ${ }^{a}$, Don M. Tucker a, Marta Kutas ${ }^{\mathrm{b}}$ and Michael I. Posner ${ }^{\mathrm{a}}$ \\ ${ }^{a}$ Brain Electrophysiology Laboratory, Department of Psychology, University of Oregon, Eugene, OR (USA), \\ and ${ }^{b}$ Department of Cognitive Science, University of Califormia San Diego, La Jolla, CA (USA)
}

(Accepted for publication: 9 September 1992)

\begin{abstract}
Summary When subjects read an semantically unexpected word, the brain electrical activity shows a negative deflection at about 400 msec in comparison with the response to an expected word. In order to study the brain systems related to this effect we mapped it with a dense (64-channel) electrode array and two reference-independent measures, one estimating the average potential gradients and the other radial current density. With these measures, the event-related brain potential (ERP) begins at about 70 msec with the P1, reflecting bilateral current sources over occipitoparietal areas. A strongly left-lateralized N1 then follows, peaking at about $180 \mathrm{msec}$, accompanied by an anterior positivity, the P2. A separate posterior positive pattern then emerges that seems to repeat the topography of the P1. Next, at about $350 \mathrm{msec}$, the ERP for the congruous word develops a P300 or LPC, characterized by a diffuse positivity over the superior surface of the head and several negativities over inferior regions. This superior source/inferior sink pattern of the LPC is greater over the left hemisphere. In contrast, the ERP for the incongruous word in this interval displays the N400 as a period in which topographic features are absent. At about 400 msec the ERP for the incongruous word begins to develop an LPC, which then remains relatively symmetric over the two hemispheres.
\end{abstract}

Key words: VEP; P1; P100; N1; N170; P2; P200; P3; P300; N4; N400; LPC; Topography; Semantic priming; Lexical access

Event-related brain potentials have been useful in studying the cognitive processes of language. One line of research has explored the electrophysiological responses of the brain to violations of semantic context, The N400 is a negative deflection in the event-related potential about $400 \mathrm{msec}$ after stimulus onset that was originally observed when subjects read sentences that ended with semantically incongruous words (Kutas and Hillyard 1980b). This effect was found to be sensitive not only to semantic incongruity, but to more subtle variations in semantic constraint as well. The amplitude of the $\mathrm{N} 400$ is smaller when the sentence ending is less expected, apparently varying with expectation in a continuous fashion (Kutas and Hillyard 1984; Kutas et al. 1984). Other studies have shown that the N400 is not restricted to the final word of a sentence. For example, words appearing toward the beginning of a sentence elicit larger N400s than later words (Kutas and Van Petten 1988; Van Petten and Kutas 1990).

Correspondence to: Don M. Tucker, Department of Psychology, University of Oregon, Eugene, OR 97403 (USA).

\footnotetext{
* This research was supported by NIMH Grants MH42129 and MH42669 and by a grant from the Pew Memorial Trusts and the James S. McDonnell Foundation to support the Center for the Cognitive Neuroscience of Attention.
}

Findings such as these suggest that the N400 may reflect the degree to which a word is expected within the current semantic context.

Evidence on the anatomic substrates of the N400 has come from studies of surgically brain-lesioned patients, intracranial recordings, and magnetoencephalographic (MEG) recordings. Kutas et al. (1988a) presented congruous or incongruous terminal words to the left, right, or bilateral visual fields of commissurotomy patients following binaural presentation of sentence contexts. All patients showed an N400 to incongruous words presented to the right visual field (left hemisphere). When presented to the left visual field, only those patients with evidence of right hemisphere linguistic capabilities responded to incongruity with an $\mathrm{N} 400$. Interestingly, the scalp distribution of the $\mathrm{N} 400$ did not vary significantly with the visual field of presentation. Kutas et al. (1988a) proposed that, among other possibilities, a left deep medial source for the $\mathrm{N} 400$ could account for this pattern of results.

Recordings of the ERP from intracranial electrodes have also suggested a left-temporal generator for the N400. ERPs recorded intracranially from medial temporal structures have been found to be sensitive to the congruity of sentence endings (McCarthy and Wood 1984) and to lexical and repetition effects with single word presentations (Smith et al. 1986). These intracra- 
nial findings are thought to be related to the scalp-recorded N4/P3 complex that has been shown to be sensitive to word repetition (Halgren and Smith 1987).

Whereas ERP methods rely on recordings of voltage at the scalp, the MEG measures the magnetic field that is perpendicular to the flow of current, thus providing unique information on neural generators. Schmidt et al. (1989) recorded both MEG fields and scalp electrical potentials while subjects read sentences with congruous and incongruous endings. Like the scalp potentials, the MEG responses differed with the congruity of the sentence. Mapping of the neuromagnetic N400 was consistent with a left mid-temporal generator.

\section{Topographic analysis}

Mapping scalp electrical recordings of the $\mathrm{N} 400$ has also provided information on the relation of this component to underlying brain activity, and to other components of the ERP. The scalp distribution of the N400 is typically described as widespread, but some regional differences have been observed. With an ear or mastoid reference electrode, the amplitude of the N400 is usually found to be maximally negative at centroparietal sites. Although the N400 is distributed bilaterally, most studies have found it to have greater amplitude and duration over the right than left hemisphere (Kutas and Hillyard 1982; Kutas and Van Petten 1988). In some cases, this asymmetry was more pronounced in right-handed subjects with no familial left-handedness (Kutas and Van Petten 1988). These topographic effects were robust across differences in presentation rate and differences in the proportion of congruous to incongruous sentences (Kutas and Van Petten 1988).

As well as suggesting candidate neural mechanisms, topographic evidence is also relevant to the relationship between the N400 and other ERP components. Kutas and her colleagues have emphasized that the $\mathrm{N} 400$ is sensitive to semantic processing constraints (Kutas and Van Petten 1988). Other researchers have claimed that the $\mathrm{N} 400$ may be a later manifestation of the $\mathrm{N} 2$ that is normally associated with physical incongruities (Polich 1985b; Herning et al. 1987), and some findings have suggested that both the N2 and the N400 appear in the ERP because of the delay of the P300 caused by increased demands for perceptual or semantic processing (Polich 1985a,b; Deacon et al. 1991). Traditionally, ERP components are differentiated on the basis of their latencies, wave morphologies, and response to stimulation. An accurate analysis of the scalp topography of the $\mathrm{N} 400$ could provide important new information for component identification (Lehmann and Skrandies 1985) and thus shed new light on these issues.

A problem, however, with previous studies of the N400 is that they have analyzed electrical recordings referenced to ear or mastoid sites. With referenced data, it is not possible to determine whether the observed voltage changes are due to activity at one electrode or the other. The electrical activity of the brain is volume-conducted throughout the head (Nunez 1981). Because there are no "inactive" sites on the head, the spatial distribution and morphology of event-related potentials in most of the published literature do not reflect activity at the "active" electrode, but the difference in voltage between the active and the reference sites (Nunez 1981, 1990; Lehmann and Skrandies 1985).

One way to characterize electrical activity at a given site on the head is to compute its difference from the "average reference" (Offner 1950; Lehmann and Skrandies 1985). For each time point, the average-reference solution transforms the voltage at each electrode into a difference between the electrical activity at that electrode and the mean activity at all recording sites. It can be shown that the average-reference will approach zero if it is computed from electrodes evenly covering the entire surface of the head, because both the positive and negative fields of each dipole source would be measured equally (Bertrand et al. 1985). However, if the scalp coverage is spatially unequal, as is usually the case due to the inaccessibility of the brain's inferior surface, then the average-reference will be biased in the direction of that inequality (Tomberg et al. 1990).

Bertrand et al. (1985) suggest that the utility of the average reference would be improved by weighting the potential at each electrode according to its topographic distribution. This solution was recognized earlier by Hjorth (1975, 1980). Hjorth's method estimates the gradients in electrical potential directed at the index site from all directions. This estimate is provided by computing the voltage difference between the index site and each other recording site, and dividing this voltage by the surface distance between the index site and the other recording site. The mean of these voltage slopes or gradients from all montage sites directed toward the index site is termed the average gradient or "source derivation" (Hjorth 1980). It can be seen that this measure is similar to an average reference, except that each of the voltages entered into the reference is weighted by its distance from the index site.

Instead of Hjorth's term, "source derivation," which is suggestive of localization of the underlying electrical source, we refer to this measure as the "average potential gradient" with the symbol " $r V$ " (for radial voltage gradient). Thus, whereas a traditional referenced recording requires a consideration of two electrode locations on the head ("active" and "reference") for an accurate interpretation, the rV estimate is meaningfully attributed to one site and is, therefore, useful for topographic studies.

Another measure that is useful for topographic studies is radial current density (Perrin et al. 1989). In this 
approach, current density is computed from the curvature of the interpolated voltage surface. The electrode sites on the head are projected onto the surface of a unit sphere. The mathematical splines describing the sphere's surface are fit to the voltage values at the multiple sites and interpolated to approximate the continuous voltage distribution across the surface. The 2-D Laplacian of the spherical splines can then be computed to yield the second spatial derivative of the voltage surface, indicating radial current density at each point on the surface.

Hjorth's average gradient estimate is computed from measured data points, whereas the Perrin et al. current density estimate is computed from the interpolated voltage surface. Because it is computed from discrete points, the average gradient computation may be distorted when there is not an equal coverage of the head surface. On the other hand, the Perrin et al. method requires that the interpolation method be highly accurate, if its second derivative is to be an accurate and stable index of local current density.

Because it describes the mean voltage gradient over distance, the Hjorth measure is a similar metric as the traditional referenced voltage recording, which also describes the difference in potential (and thus the gradient) between two locations on the head. This metric similarity may be important in relating reference-independent $\mathrm{rV}$ data to the familiar ear- or mastoid-referenced ERPs in the literature. In contrast, by describing the 2-D Laplacian or second derivative of the voltage surface, the current density measure is sensitive not to voltage slopes, but only to changes in slope (curvature). Therefore the current density measure highlights focal current sources and sinks, but it may not show the general terrain of the scalp electrical fields that are seen in traditional voltage recordings.

In the present research we examined the visual ERP during word reading with conventional mastoid-referenced data, with Hjorth's estimate of average potential gradients (rV), and with the Perrin et al. method of computing radial current density (rCD). To provide an electrode array dense enough for an accurate mapping of electrical fields across the entire head, we developed the 64-channel geodesic electrode net. This device arranges simple $\mathrm{Ag} / \mathrm{AgCl}$ sponge electrodes to provide an even geometric distribution of electrodes across the scalp superior to the canthomeatal line (Fig. 1).

\section{Method}

Thirty-six right-handed, native English-speaking, University of Oregon undergraduates participated in the experiment for course credit. Following application of the electrode net $(30-60 \mathrm{~min})$, the subject entered a sound-attenuated booth and was seated approximately
$70 \mathrm{~cm}$ (eye to screen) from the monitor upon which stimuli were presented. The subject was asked to relax with closed eyes while 30 continuous 2 sec epochs of resting EEG were collected. Next the subject was told: (1) he or she would see a series of sentences presented one word at a time, (2) some of the sentences would end with a semantically congruous word and others would end incongruously, (3) to blink between sentences and between the first couple of words in any sentence, but not during the end of the sentence when the electrophysiological data were being recorded.

The subject completed a block of 10 practice trials ( 5 congruous and 5 incongruous), then 4 blocks of experimental trials ( 15 congruous and 15 incongruous). The presentation order of the sentences was determined randomly beforehand within each block, but no more than 3 sentences in the same condition appeared consecutively. The same presentation order was used for each subject.

Each sentence began with a central fixation mark (a plus sign) for $900 \mathrm{msec}$. The words appeared one at a time in the center of the screen. Words ranged from 3 to 13 letters long subtending a visual angle of $0.8-4.3^{\circ}$. All words were presented in lower case letters. Each word, including the final word, was on for $105 \mathrm{msec}$ then was immediately replaced by the fixation mark. The inter-word interval was $900 \mathrm{msec}$. A period was appended to the final word of each sentence, denoting the end of the sentence. EEG collection began 180 msec before the onset of the terminal word and lasted for $2 \mathrm{sec}$. The fixation mark remained on the screen for $1725 \mathrm{msec}$ after the offset of the terminal word. The inter-sentence interval was approximately $4800 \mathrm{msec}$. Words were presented in 20-point bold Geneva font.

After each block of trials the subject was given a recognition questionnaire. The questionnaire showed all but the final word of 26 sentences. Ten sentences were new (not previously presented) and the remaining sixteen were presented in the previous block ( 8 congruous and 8 incongruous). The subject was instructed to circle the sentences that were presented in the previous block. After the recognition questionnaire the subject rested before beginning the next block of trials.

The experimental stimuli were 120 sentences selected from those used by Bloom and Fischler (1980) and Kutas and Hillyard (1980b). Half of these were transformed into semantically incongruous sentences by substituting an inappropriate word for the original ending. Congruous and incongruous words were matched in length and frequency of occurrence in the English language (for congruous, mean length $=4.8$, median frequency $=100$ occurrences /million; for incongruous words, mean length $=5.8$, median frequency $=104$ occurrences $/$ million). Congruous and incongruous sentences ranged in length from 5 to 11 words (congruous, mean number of words $=7.85$; incongru- 
ous $=7.93$ ). Congruous endings were also coded according to their cloze probabilities, the degree to which they were predicted by the semantic context of the sentence (Taylor 1953); approximately half the words were of low cloze probability $(P=0.20-0.50)$ while the other half were of high cloze probability $(P=0.60$ 0.90 ). Care was taken to insure that no content words were repeated within or between these 120 sentences. Forty distractor sentences for the recognition test were also chosen from Bloom and Fischler (1980).

\section{ERP recording}

The EEG was recorded from a montage of 64 recording sites with the geodesic electrode net (Tucker submitted). This device is a network of $\mathrm{Ag} / \mathrm{AgCl}$ saline sponge electrodes, each held in a plastic tube. The tubes are arranged by elastic threads in a geodesic pattern, in which the surface of a sphere is partitioned into equal triangles. The geodesic geometry, implemented with the elastic tension network, is adjusted to conform a spherical surface to the shape of an average sized head. When applied, it further conforms to the unique geometry of the subject's head. The net is oriented with the central vertex of the upper icosohedron of the geodesic geometry placed at the adjusted vertex of the skull $(\mathrm{Cz}$ measured not from the inion but from where the extension of the canthomeatal line crosses the posterior midline), and the perimeter of the outer ring of electrodes is placed on the canthomeatal line (from the external canthus to the auditory meatus). The front ring of electrodes (sites 12, 9, 5, and 48) are placed above the eyebrows, and the perimeter is positioned on the side at the left and right mastoid bones (sites 24 and 65). As with the measurements of the international 10-20 system, these procedures are adequate to replicate the electrode placements on a given head. However, the variability in individual heads with respect to brain structures is still substantial. Given these practical constraints, the geodesic tension structure provides an even surface distribution of the electrodes, and an even conformation of the spherical geodesic structure to actual head geometries, suitable to the surface interpolation provided by spherical splines (Perrin et al. 1989).

Fig. 1 shows the distribution of electrodes on the schematic 2-D projection used for portraying the ERP topography. In addition to the sponge electrodes of the net, external $\mathrm{Ag} / \mathrm{AgCl}$ electrodes were attached with double-sided adhesive tape for the nasion, external canthus, infraorbital, and left and right mastoid sites. The right mastoid was the reference for all recording channels. A ground electrode was placed at the position marked "G." The 2-D locations of the electrodes in Fig. 1 and in the color maps in this paper were determined by projecting the 3-D locations of the electrodes onto the 2-D surface. The net was applied

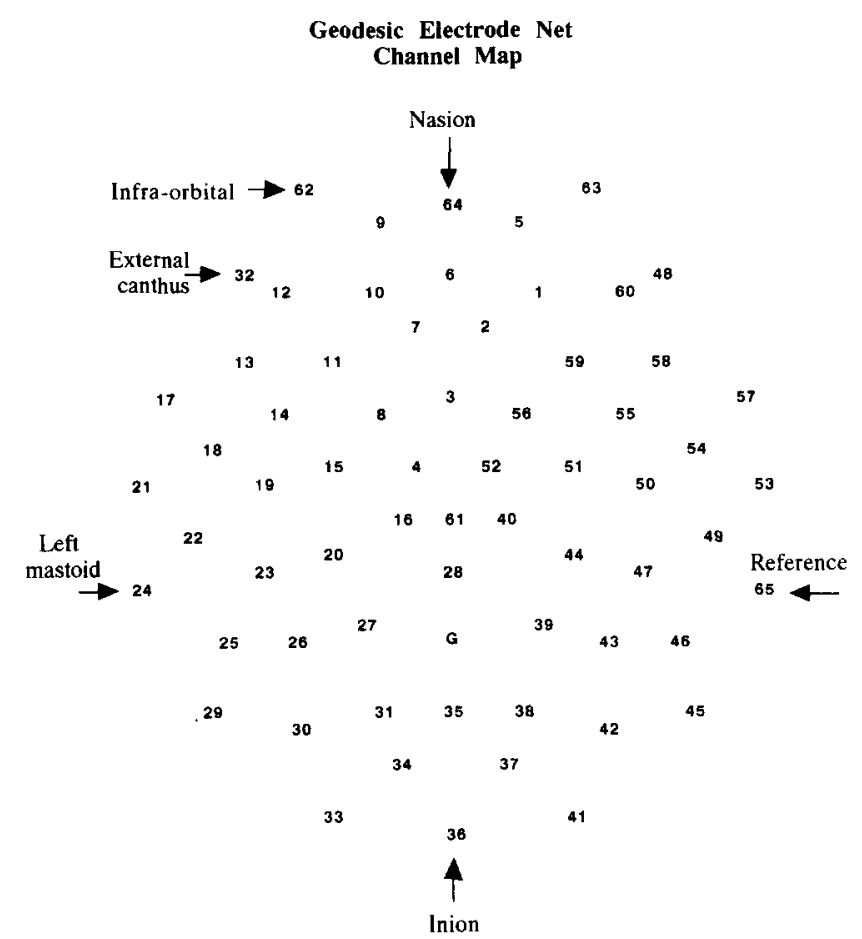

Fig. 1. Diagram of channel locations on the geodesic electrode net. Each number represents a recording site.

to the model head, and the electrode positions marked. The polar coordinates (angle and radial distance from the vertex) of each electrode site were then used to project that site's location onto the 2-D surface of Fig. 1.

The sponge electrodes of the geodesic electrode net provide impedances in the range of $10-40 \mathrm{k} \Omega$. This range is adequate for amplifiers with high $(>100 \mathrm{M} \Omega$ ) input impedances, such as those used in the present study. After amplification with a $0.1 \mathrm{~Hz}$ to $50 \mathrm{~Hz}(3 \mathrm{~dB}$ attenuation) bandpass and a $60 \mathrm{~Hz}$ notch filter, the signals were digitized with a 16 -bit A/D converter at a rate of $125 \mathrm{c} / \mathrm{sec}$ and stored to computer memory.

For each subject, each $2.048 \mathrm{sec}$ (256 sample) 64channel epoch of the EEG was examined off-line to reject eye movement, blink, movement, or other noncephalic artifacts. The data were then averaged for each experimental condition (congruous and incongruous endings) and calibrated for amplifier zero and gain levels.

The initial net prototypes used in this experiment suffered from electrode polarization on several occasions. Because of this and other technical problems, 6 subjects had poor data on most or all channels; the data from these subjects were rejected at the outset as unusable. Seven additional subjects were eliminated from the analysis, 6 because of artifactual data from more than 8 channels and 1 because of an unusual handedness report (she said she was forced to be left-handed at an early age). For each remaining sub- 
ject, the artifactual channels were noted and exempted from the artifact screening for that subject. The data from these channels were omitted in the computation of the grand averages and were entered as missing data in the ANOVAs. Of the 23 right-handed subjects retained for the analysis, 6 had no bad channels, 10 had 1,3 had 2, 1 had 4, 1 had 5, 1 had 7, and 1 had 8 artifactual channels. Six of these subjects reported left-handed parents or siblings. All recordings used a right mastoid reference. They were re-referenced algebraically to the average voltage of the left and right mastoids to allow comparison with the typical linked ears or linked mastoid referenced data in the literature. These data are referred to as the "mastoid-referenced" data.

\section{ERP analyses}

Reference-independent derivations. To compute Hjorth's (1980) estimate of average potential gradients, the inter-electrode distances were measured from the 64-channel net's actual electrode positions when the net was positioned on an average-sized model head. A distance coefficient matrix (the inverse of the distance of each electrode from the index site scaled as a proportion of the total distances) was created with the same order as the EEG data matrix for the ERP epoch. When multiplied by the data matrix, the product was the distance-weighted reference matrix. Subtraction yielded a matrix of average gradient $(\mathrm{rV})$ estimates for each channel for that epoch. By scaling the distances as proportions of the total of the distances used for that index site, Hjorth's (1980) method adjusts for the fact that some electrode sites are closer to the center of the montage than others. The following equation, adapted from Hjorth (1980), illustrates how each distance, used to weight the potentials comprising the average reference, is expressed as a proportion of the total distances in the montage.

$\mathrm{rV}_{\mathrm{s}}=\mathrm{V}_{\mathrm{s}}-\frac{\mathrm{V}_{1} / \mathrm{r}_{\mathrm{s} 1}+\mathrm{V}_{2} / \mathrm{r}_{\mathrm{s} 2}+\ldots+\mathrm{V}_{\mathrm{n}} / \mathrm{r}_{\mathrm{sn}}}{1 / \mathrm{r}_{\mathrm{s} 1}+1 / \mathrm{r}_{\mathrm{s} 2}+\ldots+1 / \mathrm{r}_{\mathrm{sn}}}$

The average potential gradient $(\mathrm{rV})$ is computed separately for each electrode site (s). The mastoid-referenced potentials recorded at the index site (s) and all other locations $(1 \ldots n)$ are denoted by $V_{s}$ and $V_{1} \ldots V_{n}$. The distances from the index to each of the other electrodes are $r_{s 1}$ to $r_{s n}$. The metric for this $\mathrm{rV}$ measure is thus a gradient, $\mu \mathrm{V} / \mathrm{cm}$, except that the distance in the divisor was expressed for each channel not in $\mathrm{cm}$ but as a proportion of the total distances available for that site's computation.
Prior to the final matrix subtraction, an additional channel was created for the right mastoid reference electrode site by entering zeroes for each data sample. Then the subtraction regenerated the average potential gradients at the reference electrode (channel 65), defined as zero minus the distance-weighted mean voltage across the other channels, i.e., the voltage gradients directed from all other head sites toward the reference electrode. For an intuitive appreciation of this computation, consider the recording as 64 measurements of the right mastoid site, each with a different reference electrode.

An alternative reference-independent characterization of the data was computed as the average-reference derivation (Lehmann and Skrandies 1985), in which the reference for the index site is simply the mean voltage of all other head locations. In our analyses of data sets with fewer (e.g., 16) channels, the average-reference differs substantially from Hjorth's measure. This reflects the bias of the unweighted amplitude mean as a reference when the head fields are not sampled evenly (Tomberg et al. 1990). However, for the present 64channel montage, the improved sampling of the whole-head volume conduction caused the wave form plots of the average-referenced data to be almost identical to those of the Hjorth-transformed $\mathrm{rV}$ estimates, differing primarily in scale. To conserve space, the present analysis focuses on the $\mathrm{rV}$ estimates.

Topographic interpolation. To generalize the observed voltage gradients to estimate the pattern of average potential gradients across the head surface, the $\mathrm{rV}$ data from a given time sample were interpolated using spherical splines (Perrin et al. 1989). To make the spherical approximation a reasonable one, the projection of electrode sites at actual head locations to the spherical generalization must be accurate. As described above, the geodesic electrode net positions the electrodes by conforming the spherical geodesic geometry to individual head shapes. To project the actual head locations onto a spherical model, the polar coordinate measurements (from the vertex) of the electrode locations on the head model were projected into spherical coordinates. The angle and surface radius of each electrode from the vertex were used to locate the position of the electrode on the sphere. After spherical interpolation, this translation was used to project 2-D images of the head surface from the data at the spherical spline coordinates.

Radial current density computation. From the spherical interpolation of the voltage surface, the pro-

Fig. 2. Grand average of mastoid-referenced voltage from 23 right-handed subjects. Each axis represents a single channel location and roughly corresponds to the spatial layout depicted in Fig. 1. The scale of the axes is shown in the lower, midline area of the figure. Positive is up. The thin line is the ERP to the congruous ending word and the thick line is the ERP to the incongruous word. The vertical line on the graph is at stimulus onset (184 msec). 


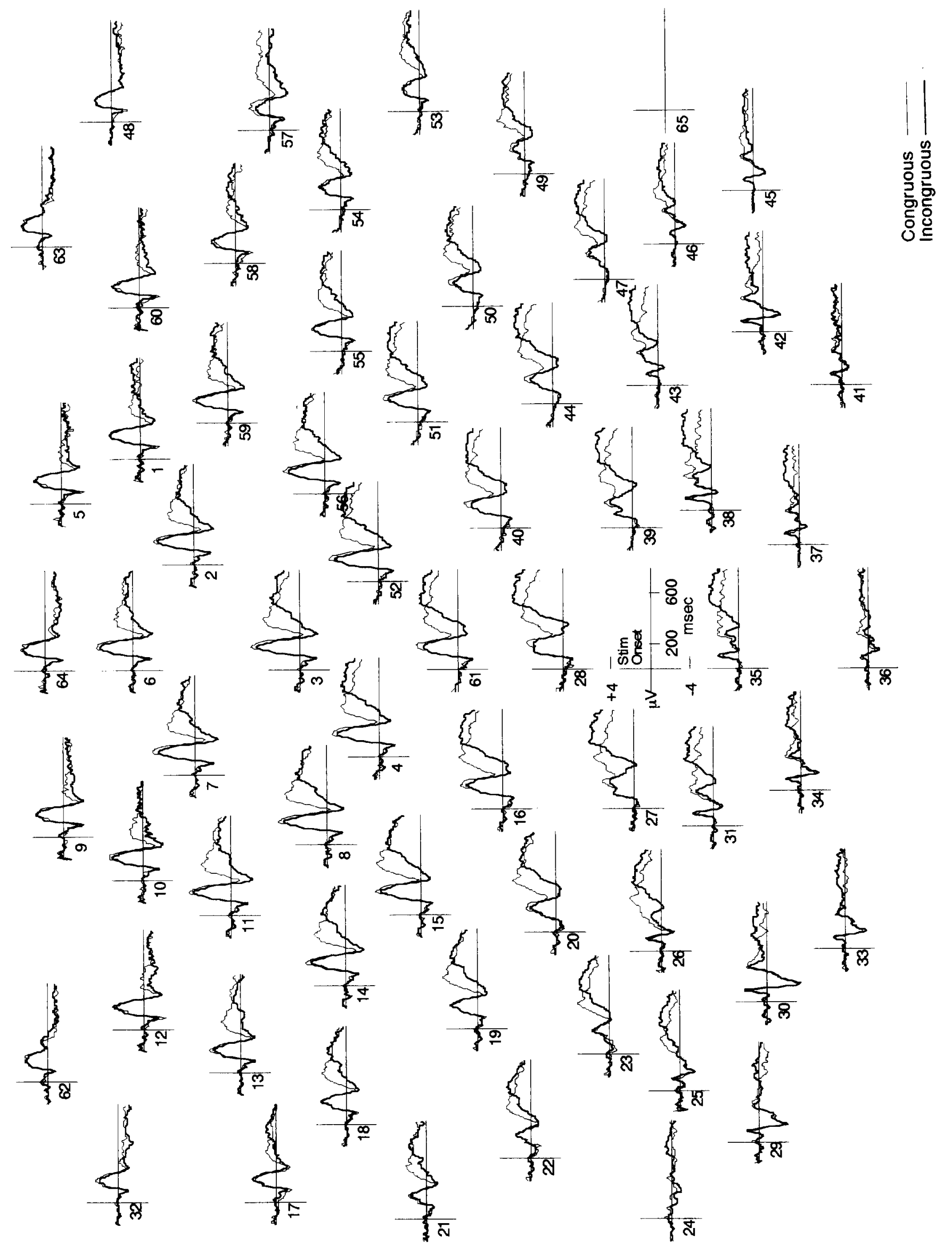


cedures described by Perrin et al. (1989) yield the 2-D Laplacian of the voltage surface characterizing radial current density (rCD). This measure was computed from the initial (right-mastoid-referenced) voltage (rather than rV) data, with zeroes entered at the right mastoid location.

\section{Results}

The presentation of this extensive set of data is necessarily selective. The difference between the mastoid-referenced and the reference-independent (rV) topography is illustrated by wave form plots. The statistical analysis with ANOVA focuses on the semantic congruity effect within selected windows of the $\mathrm{rV}$ wave forms. In addition, for the $\mathrm{N} 400$ window, the mastoid-referenced data are also analyzed in the ANOVAs to verify replication of the semantic effect described in the literature. Topographic images are shown for the reference-independent methods ( $\mathrm{rV}$ and rCD) which provide accurate estimates of the electrical activity at specific head locations. For the rV measure, the 2-D topographic images illustrate the effects examined in the ANOVAs. For each of these $\mathrm{rV}$ images (Figures 4-10), the corresponding $\mathrm{rCD}$ image is presented for comparison. However, given the exploratory nature of the $\mathrm{rCD}$ effects, these data were not analyzed statistically.

In naming the ERP features in the animated maps, we have tried to relate these features to well-known ERP "components" identified from wave peaks. Examining ERP waves, components have been variously defined in the literature in terms of the sequence of the bump on the wave (N1, N2, etc.) or the latency associated with it (N170, N283). In the following descriptions, both for the wave form plots and the animated maps, we attempt to identify events as patterns in the animated maps that are coherent in both spatial extent (topography) and time course. We then relate these to conventional "components" by referring to sequence numbers early in the wave, where these seem discernible (N2), and to latencies later in the wave, where these seem more descriptive (N400). By mixing these naming conventions we emphasize that a component definitional scheme based on inspection of individual channels is inadequate to describe the spatial and temporal patterning of electrical events examined across the whole head.

\section{Wave form plots}

The mastoid-referenced grand averages for the 23 subjects are shown in Fig. 2. These mastoid-referenced plots show the typical N400 effect seen in the literature, particularly for the central and parietal channels. After the N1/P2, the ERP to the incongruous word diverges in a negative direction, beginning at about 250-300 msec, and maintains this relatively negative deflection for 200-300 msec. The data from this 64channel array show substantial differentiation between semantic conditions among posterior locations, but less regional differentiation among central and anterior channels.

The rV estimates with Hjorth's method are shown in Fig. 3. There are two major factors producing the differences between these data and the mastoid-referenced plots. One is the use of distance-weighting, with the effect of emphasizing local changes more than long-distance voltage effects. Because the $\mathrm{rV}$ plots are quite similar to the average reference plots, this factor does not seem to have a major effect in the present data. The second factor is the mastoid-referenced data's inherent confounding of electrical potentials at the "active" site with those at the reference site. This factor has a substantial effect, as shown by the size of the amplitude changes in the mastoid wave forms in Fig. 3, either the "recorded" left mastoid of channel 24 or the "regenerated" right mastoid at location 65 .

Thus much of the apparent ERP wave form in the mastoid-referenced data of Fig. 2 actually represents the inverted polarity of the potentials occurring at the mastoid locations. The accuracy of the data for the mastoid channels in the $\mathrm{rV}$ wave forms in Fig. 3 is supported by the similarity to wave forms from nearby locations, particularly those from inferior posterior sites (e.g., 29, 25 on the left; 45,46 on the right). The contribution of the potentials at the mastoid to ERP components in the mastoid-referenced data occurs throughout the ERP epoch, influencing the P1, N1, P2 and later components.

The differentiation between semantic conditions is clearly apparent in both Figs. 2 and 3, but the localization of the effect differs importantly depending on the reference assumption. Whereas the N400 effect appears to be located over the superior central and parietal areas in the mastoid-referenced plots (Fig. 2), it is shown in the $\mathrm{rV}$ plots to be divided between a superior negativity and an inferior positivity.

Because it is a difference effect between semantic

Fig. 3. Grand average of voltages transformed with Hjorth's distance-weighted average reference indicating average potential gradients (rV) from 23 right-handed subjects. Each axis represents a single channel location and roughly corresponds to the spatial layout depicted in Fig. 1. The scale of the axes is shown in the lower, midline area of the figure. 


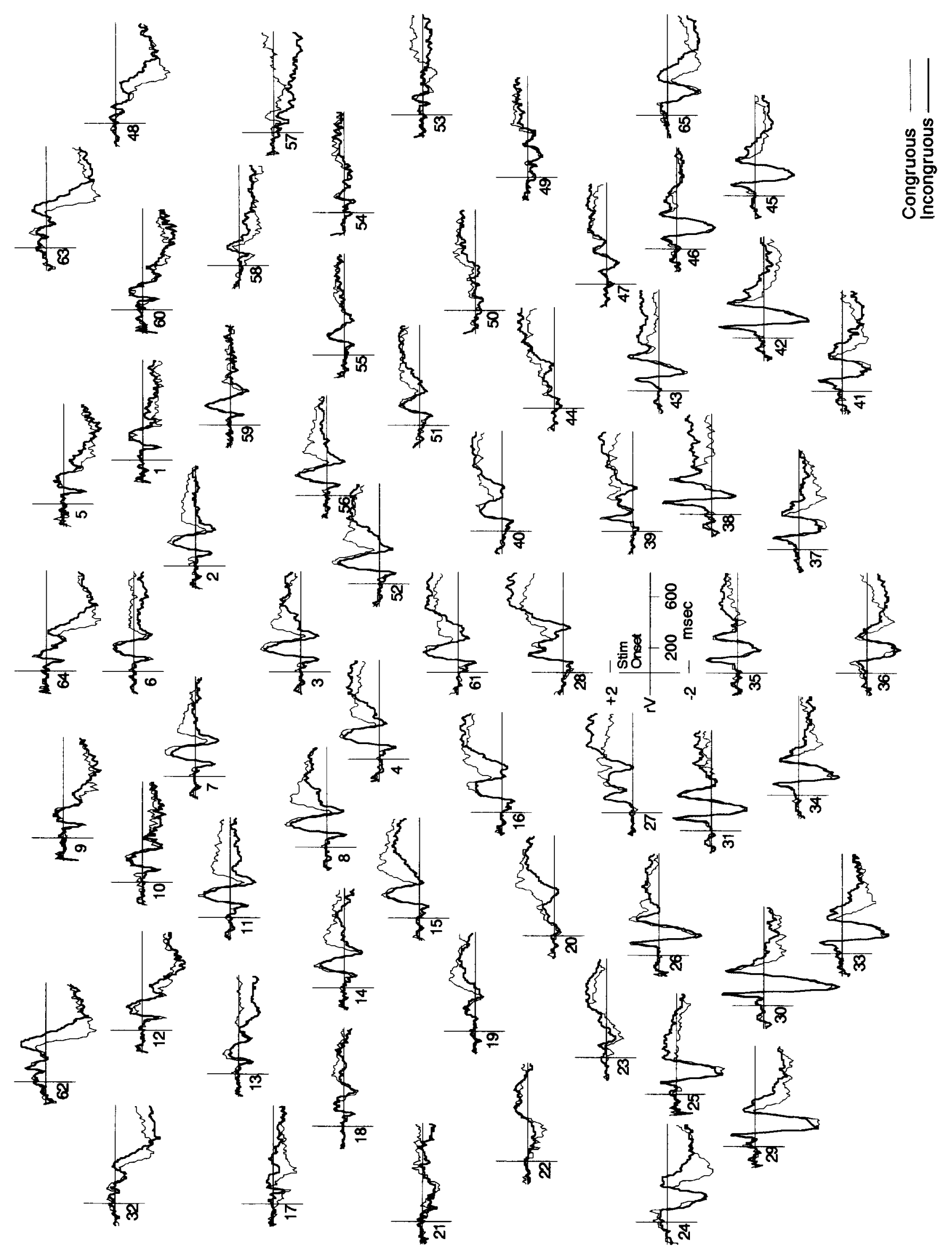


conditions, the $\mathrm{N} 400$ phenomenon must be understood in terms of the ongoing activity in the ERPs from which it is derived. In Fig. 3, it can be seen that beginning at about $300 \mathrm{msec}$ for the congruous word there is a strong positive deflection over superior scalp regions and a negative deflection over inferior regions. This appears to be the $\mathrm{P} 300$ or late positive complex (LPC). We have observed a similar superior/inferior source/sink pattern for P300s in other recent 64-channel data sets examined with the $\mathrm{rV}$ derivation in our laboratory. As with the LPC in the present data, the regions that show the greatest negativity (current sink) during the LPC are the most inferior locations, including the infraorbital $(62,63)$, mastoid $(24,65)$, and inferior posterior $(29,30,33,41,42,45)$ channels that have not been examined in previous topographic studies of the P300 or LPC. Clearly, the definition of the P300 as a positivity has occurred because the "active" sites were on the superior surface of the head and the ear or mastoid reference electrode was assumed to be inactive.

Once the bipolar nature of the LPC is recognized in Fig. 3, the N400 difference wave can be understood in terms of the appearance of this LPC for the congruous word at about $300 \mathrm{msec}$ and the corresponding lack of this LPC for the incongruous word. The congruous word LPC shows a strong positive peak over left and medial central superior locations (e.g., 20,15,8,3) and a negative-going wave in the inferior channels in both posterior and anterior regions of the head. For the superior sites, the incongruous word ERP shows the absence of the positive-going LPC in the $300-500 \mathrm{msec}$ range, but after $500 \mathrm{msec}$ a strong LPC has developed for that word as well.

For the inferior sites, the situation is somewhat more complex. In the anterior inferior sites, particularly the infraorbital $(62,63)$ and external canthus $(32$, 48) electrodes, the negative wave (current sink) for the incongruous word appears to lag that for the congruous word. But for inferior posterior sites, particularly on the left side $(24,29,33)$, the incongruous word ERP continues in a positive direction out of the extended $\mathrm{N} 1$ trough at about $300 \mathrm{msec}$, whereas this second positive peak for the congruous word has apparently been attenuated by the negative-going inferior wave of the LPC.

\section{Animated topography}

Although the wave form plots provide useful information on the time course of the ERP at each of the measured locations, it is difficult to discern the spatial patterning of the electrical field across head regions at any one point in time by looking at these plots. Similarly, examination of a map of the electrical fields at one point in time fails to convey the dynamic temporal course that shows fields appearing or disappearing in certain brain regions synchronously. To visualize both time course and topography, we created animated maps. For each sample of data, an interpolated surface was created with spherical splines of the 65-channel $r V$ data. The series of maps describing the ERP epoch were animated on the graphics screen. A parallel animation of radial current density (rCD) was created with the 2-D Laplacian computation from the spherical splines on the voltage data.

Several key samples from the animations were selected to illustrate the major differences between the ERPs for the congruous and incongruous sentence endings (Figs. 4-10 for $\mathrm{rV}$ and $\mathrm{rCD}$ ). The forehead is at the top of the image, and the left hemisphere is on the left. The palette for the $\mathrm{rV}$ images is calibrated to a data range that encompasses most of the grand average voltage gradient ERPs: -2.5 to $+2.5 \mu \mathrm{V}$. Where the data exceeded this data range the palette is clipped to black or white. In addition to the raster palette, contours are plotted every $0.25 \mu \mathrm{V}$. The dots on the images show the locations of the 63 electrode locations that appear in this display space (the infraorbital channels were used in the spherical interpolations but their projected locations are outside the display space). In

Figs. 4-10. Selected frames from the rV and rCD animations for the congruous and incongruous words. The palette for these images, shown in the color bar at the right, is calibrated with the lower (black) end at $-2.5 \mu \mathrm{V}$ and the upper (white) end at $+2.5 \mu \mathrm{V}$, with values exceeding these ranges (in Figs. 5 and 10 ) pinned to black or white.

Fig. 4, $104 \mathrm{msec}$. The tight bilateral foci of the P1. This feature has emerged from a slightly more right inferior origin to focalize on these sites before appearing to move medially and superiorly to form the anterior $\mathrm{P} 2$.

Fig. 5, 176 msec. The N1/P2 complex. Note the broad distribution of the central-frontal $\mathrm{P} 2$ in the rV derivation in contrast with the more focal positivity adjacent to the N1 characterized by the second derivative function of the 2-D Laplacian on the spherical splines for the rCD plots.

Fig. 6, 312 msec. Reprise of the P1. As the P2 wanes, it appears to move back on the head and recruit the same tight bilateral foci of the P1. The P1 reprise appears to be the onset of the LPC or P 300 for the congruous word, but fades out for the incongruous word. Note the signs of frontal activity and their different appearances in the $\mathrm{rV}$ and $\mathrm{rCD}$ images.

Fig. 7, 368 msec. The N400 interval. The ERP for the congruous word has developed an abrupt and strong LPC, while that for the incongruous word has followed the P2 and P1 reprise with an interval of minimal electrical topography. 

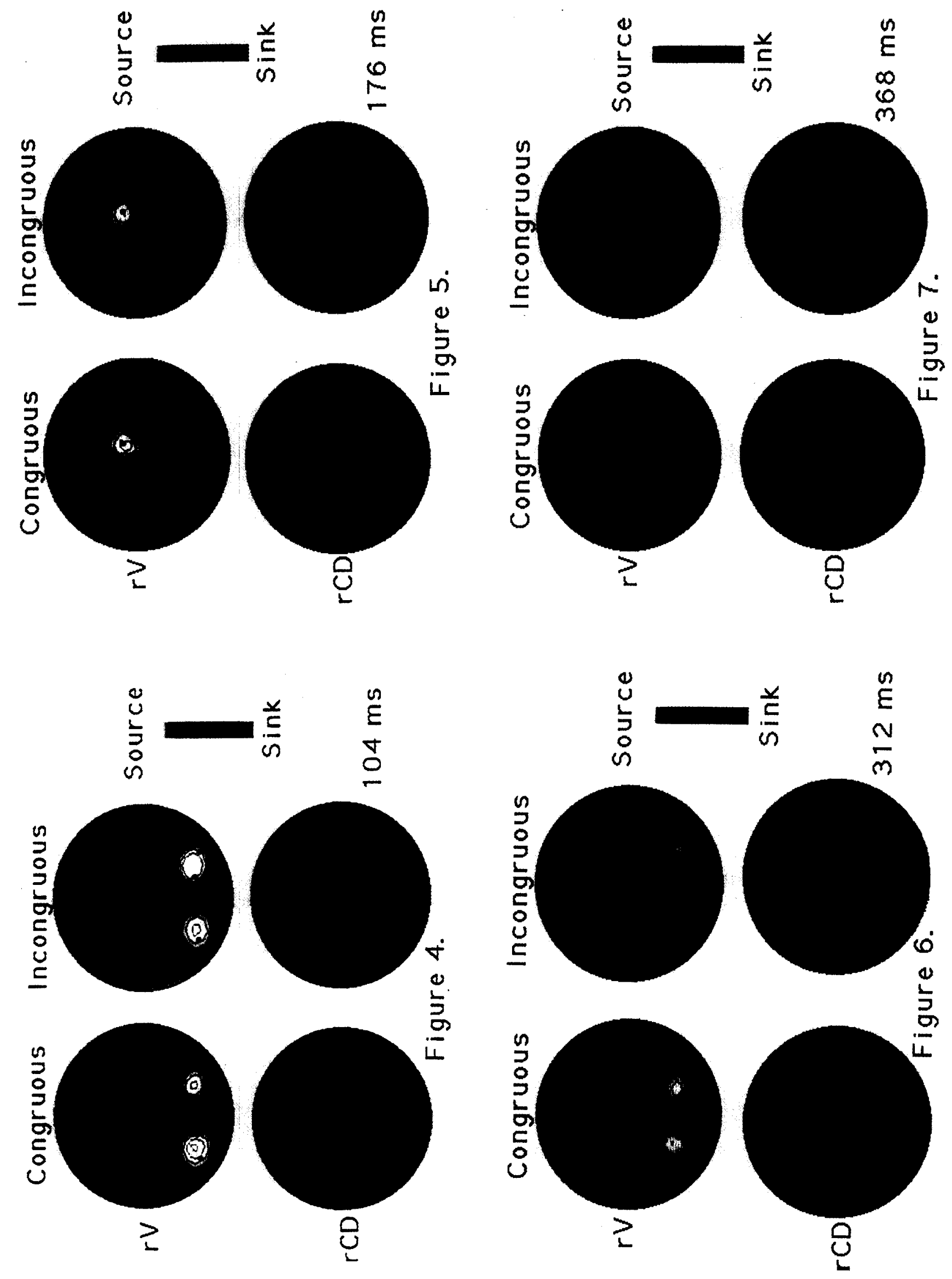
our descriptions, we focus on the $\mathrm{rV}$ images, but we attempt to point out some of the similarities with and differences from the rCD images.

The first definite features in the animations appear in the $\mathrm{rCD}$ maps by $48 \mathrm{msec}$ (after stimulus onset), while the $\mathrm{rV}$ maps still appear to be silent. The $\mathrm{rCD}$ maps show bilateral current sinks over dorsolateral frontal areas, with adjoining sources appearing to extend off the data space toward the orbital frontal areas. By the onset of the P1 (64 msec), the spherical splines localize current sources over the eyes. Certain rCD events localized to the eyes may represent retinal potentials. In addition, skull orifices at the eyes and ears provide current paths through which electrical activity of nearby brain areas may have a particularly strong influence on scalp recordings.

The first feature of the ERP appearing in both the $\mathrm{rV}$ and $\mathrm{rCD}$ animations is the P1, which is first seen as a slightly enhanced focal positivity (radial current source) on the right side at $64 \mathrm{msec}$. The P1 builds to a focal bilateral peak by $104 \mathrm{msec}$ in both the $\mathrm{rV}$ and $\mathrm{rCD}$ (Fig. 4). As is also seen in the wave form plots for $\mathrm{rV}$, the focal posterior P1s are accompanied by a diffuse anterior negativity.

After the peak of the P1, two current sinks appear immediately over the eyes in the rCD. At this time, the positivity of the P1 appears to move medially and superiorly, then frontally, until the positive region of the ERP has shifted to the central and frontal regions. This positive region seems to reflect the $P 2$ identified in the conventional mastoid-referenced ERP. As the positive maximum of the developing $\mathrm{P} 2$ crosses the vertex, the $\mathrm{N} 1$ appears as a discrete negative focus over the left inferior occipitotemporal area. The N1 peaks at $176 \mathrm{msec}$ (Fig. 5), at which time the P2 is near to its maximum as well. Although quite focal and posterior, the N1 occupies different sites from the P1. Thus, although the $\mathrm{N} 1$ may appear to be replaced by the $\mathrm{P} 2$ in wave form plots of particular channels, as topographic features the $\mathrm{N} 1$ and $\mathrm{P} 2$ are shown by the animations to be coincident phenomena.

The $\mathrm{rV}$ shows the $\mathrm{P} 2$ to be a fairly diffuse vertex positivity. Given its sensitivity to the high spatial frequencies of the potential field, the $\mathrm{rCD}$ measure resolves multiple sources within the P2 pattern (Fig. 5). One of these is adjacent to, and just superior and anterior to, the N1 sink. This source/sink combination is highly coordinated in time in the animations, giving the appearance of a tangential dipole over the left parietal area.

By 200 msec the $\mathrm{rCD}$ maps for the incongruous word show a left anterior inferotemporal current source (ch 21-17) and two sources over the eye regions that will also appear by the next sample for the congruous word. The left frontal and temporal source foci show greater and longer-lasting current density for the in- congruous than the congruous words. In addition, an especially sharp source/sink gradient appears in the left inferior frontal area for the incongruous word, perhaps reflecting the first electrophysiological differentiation between the two semantic conditions.

The N1s of other 64-channel ERPs we have observed to word stimuli have been bilateral when semantic processing was not required. In contrast, the N1 of the present data is strongly left-lateralized. The N1 is typically quite focal to this inferior occipitotemporal area, often appearing at maximal amplitude at a single channel for individual subjects. In Fig. 5, a sharp gradient can be seen to be created in the $\mathrm{rV}$ maps by the contrast of the inferior $\mathrm{N} 1$ with the superior $\mathrm{P} 2$ over the left parietal area. This is the gradient that looks like a tangential dipole in the $\mathrm{rV}$ map. For several subjects, this sharp gradient appears again over the left posterior area in the $\mathrm{rV}$ animations, as the late positive complex (LPC) develops a similar inferiornegative and superior-positive pattern.

A remarkable feature of the visual ERP seen in these maps is the reappearance of the $P 1$ pattern after the extended N1 interval. This feature can be seen in the wave form plots of the rV (Fig. 3), and it is a striking event in both the $\mathrm{rV}$ and the $\mathrm{rCD}$ animations (Fig. 6). Given that the word stimulus offset occurred 105 msec after stimulus onset, it is unlikely that this second P1 pattern at $312 \mathrm{msec}$ reflects stimulus offset. This effect occupies much the same sites as the P1, and it starts on the right side as well. We have termed this feature the "P1 reprise." As with the original P1, a number of intriguing frontal features accompany the P1 reprise. The $\mathrm{rV}$ (Fig. 6) at this point shows a dipole-like gradient over the left frontal region for both the congruous and incongruous words. However, the more focal rCD animations (Fig. 6) show the current density to be more differentiated over the left frontal region for the congruous word and more over the right frontal region for the incongruous word.

Up to this point, the ERP to the two words has been generally similar. Next, the animations show the P1 reprise to follow the same pattern as the $P 1$, moving medially and superiorly. At about $350 \mathrm{msec}$ the two ERPs diverge. For the congruous word the superior positivity develops directly into the LPC, with an initial left central predominance, but soon evidencing the broad superior positivity and inferior negativity that characterize the LPC topography. For the incongruous word, in contrast, the $\mathrm{P} 1$ reprise fades into a period in which the electrical terrain appears featureless (Fig. 7).

Both the LPC developing at this time for the congruous word and the contrasting electrical silence for tîe incongruous word are broadly distributed characteristics of the scalp electrical fields, and thus they are poorly characterized by the second-derivative, $\mathrm{rCD}$ method (Fig. 7). However, the $\mathrm{rCD}$ maps are sensitive 
to the local variations of the LPC current flow in the more inferior regions, and by $400 \mathrm{msec}$ they are beginning to resolve these local gradients into more specific patterns than the broad superior-source and inferiorsink pattern of the LPC in the rV maps. As will be characteristic of the LPC throughout the remainder of the epoch, the rCD maps delineate current sources in the inferior temporal areas (channels 21, 17 and 53, 57). In addition, both the rV and ICD maps show a focal sink over the inferior right frontal area for the congruous word (Fig. 7). This right frontal sink seems to signal the onset of the broad LPC, and it remains active throughout the congruous LPC.

As can be seen in the wave form plots, the LPC for the congruous word extends for several hundred milliseconds, waning only gradually. After its interval of flat topography, the incongruous word ERP develops an LPC, building gradually from about $400 \mathrm{msec}$ on. As the incongruous ERP begins its belated LPC, it seems to start with another $P 1$ reprise, visible at $408 \mathrm{msec}$ in Fig. 8. This effect is only right-sided and does not have the sharp circular boundaries of the P1 and the initial $P 1$ reprise. But it seems to reflect a second recruitment of the P1 pattern, at least on the right side, as the LPC is initiated for the incongruous word.

As the LPC develops for the congruous word, the $\mathrm{rV}$ maps show the inferior activity as a diffuse ring of negativity that is particularly strong in right frontal and left posterior areas (Fig. 9). The rCD derivation focalizes this activity to bilateral sources near the eyes and just anterior to the ears. Although the location of these sources is likely biased by the nearby skull orifices, there is a clear trend in the rCD animations for a left-lateralization of the anterior temporal source during the peak of the congruous LPC (Fig. 9).

Certain features of the incongruous LPC parallel those described for the congruous LPC, but with the opposite laterality. In contrast to the left-lateralization of the temporal activity associated with the congruous LPC; the anterior temporal $\mathrm{rCD}$ source during the incongruous LPC is exclusively right-sided (Fig. 10). The incongruous LPC also shows a left frontal source/sink gradient that mirrors a similar right frontal pattern seen throughout the congruous LPC.

In the next several hundred msec, the LPC for the congruous word wanes gradually, while that for the incongruous word remains strong. The incongruous LPC eventually becomes stronger than that for the congruous word (Fig. 3). The superior centroparietal positivity of the incongruous LPC seems to be more focal than the diffuse pattern seen during the congruous LPC (Fig. 10).

\section{ANOVAs}

Separate analyses of variance were performed on measures of the ERP for 5 latency regions of the ERP
TABLE I

\begin{tabular}{llll}
\hline \multicolumn{2}{l}{ Spatial factors } & \multicolumn{2}{l}{ Channels } \\
\cline { 1 - 2 } Caudal & Lateral & Vertical & \\
\hline Anterior & Left & Inferior & $9,12,17,21,32,62$ \\
Anterior & Left & Mid-vertical & $7,10,11,13,14,18,19$ \\
Anterior & Left & Superior & $4,8,15$ \\
Anterior & Right & Inferior & $5,48,53,57,60,63$ \\
Anterior & Right & Mid-vertical & $1,2,50,54,55,58,59$ \\
Anterior & Right & Superior & $51,52,56$ \\
Posterior & Left & Inferior & $24,29,33$ \\
Posterior & Left & Mid-vertical & $22,23,25,26,30,31,34$ \\
Posterior & Left & Superior & $16,20,27$ \\
Posterior & Right & Inferior & $41,45,65$ \\
Posterior & Right & Mid-vertical & $37,38,42,43,46,47,49$ \\
Posterior & Right & Superior & $39,40,44$ \\
\hline
\end{tabular}

epoch. The latencies of these windows were chosen to reflect the major features of the ERP wave forms. Three early latency windows were chosen from inspection of the posterior channels: the P1 (64-120 msec post-stimulus), the N1 (136-184 msec), and the P2 (184-248 $\mathrm{msec})$. An intermediate latency window (280-304 msec) was fitted to the difference between congruous and incongruous sentences, peaking at approximately $400 \mathrm{msec}$ in the centroparietal channels. A late latency window $(544-840 \mathrm{msec})$ was chosen to capture the late differences between congruous and incongruous sentences. The dependent measure for each of these latency windows was mean amplitude.

Five factors were entered into each ANOVA. A between subjects factor was familial handedness. The primary repeated measures factor was sentence type (congruous or incongruous). The remaining 3 factors parsed the channels into the caudality, laterality, and verticality spatial dimensions. The channels used for the 12 cells formed by the crossing of these spatial dimensions are shown in Table $I$.

A single mean amplitude was obtained for each area by averaging the mean amplitudes from the channels within the area. The number of electrodes comprising each area mean ranged from 3 to 7 . Data from all channels except those on the lateral midline were included in these analyses. The probabilities reported reflect the Greenhouse-Geisser correction for heterogeneity of covariances. Analyses were performed only on the $\mathrm{rV}$ data, except for the intermediate latency window, for which the mastoid-referenced voltages were also analyzed.

\section{Mastoid-referenced data}

Intermediate latency window (280- $304 \mathrm{msec})$. Congruous sentences (mean $=1.65 \mu \mathrm{V}$ ) were significantly more positive than incongruous sentences (mean $=0.38$ $\mu \mathrm{V}), F(1,21)=20.99$, MSe (mean square error) $=$ 10.25, $P=0.0002$. The effect of sentence type interacted with both the lateral factor and the vertical 

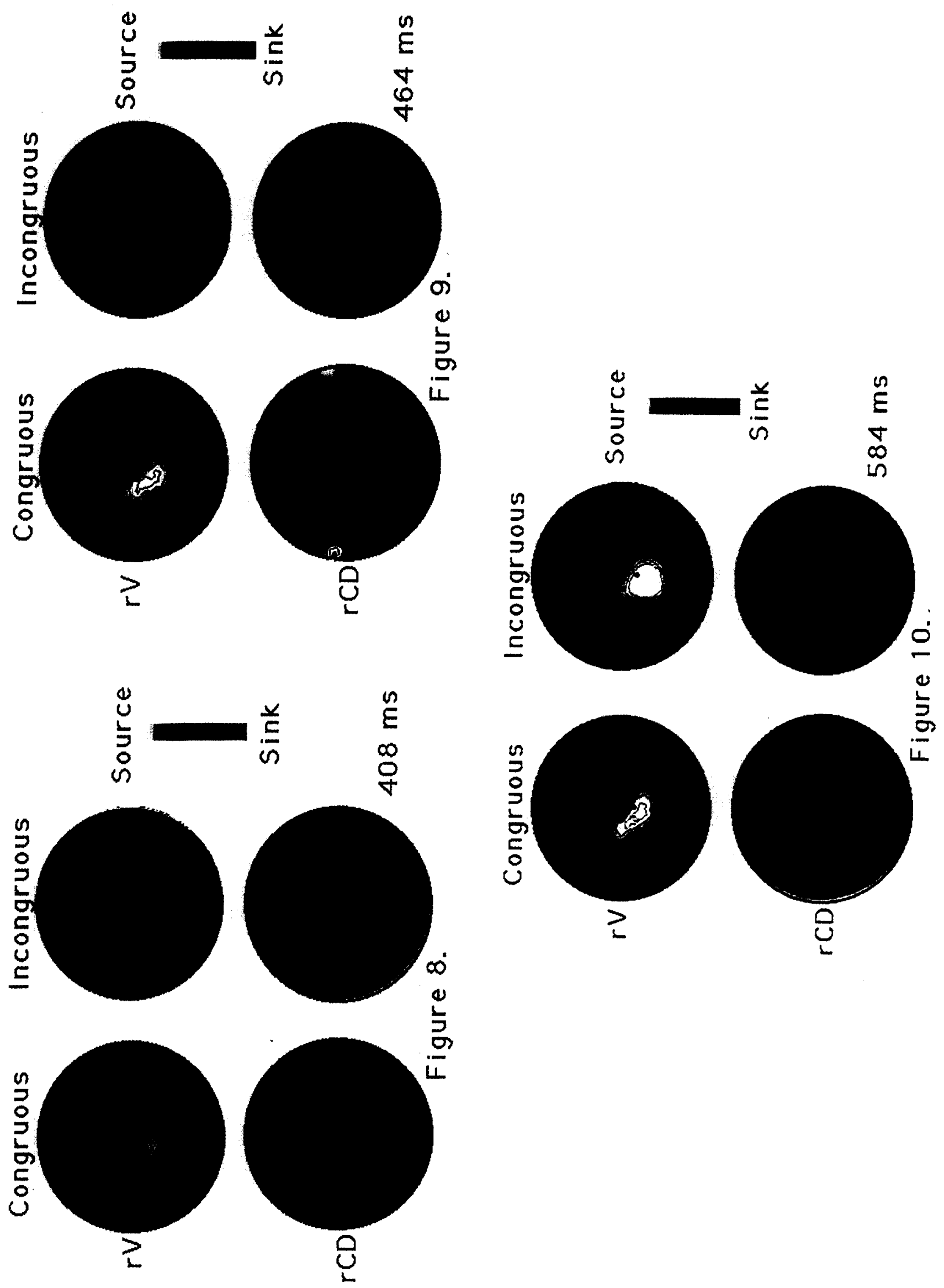


\section{Left Hemisphere}

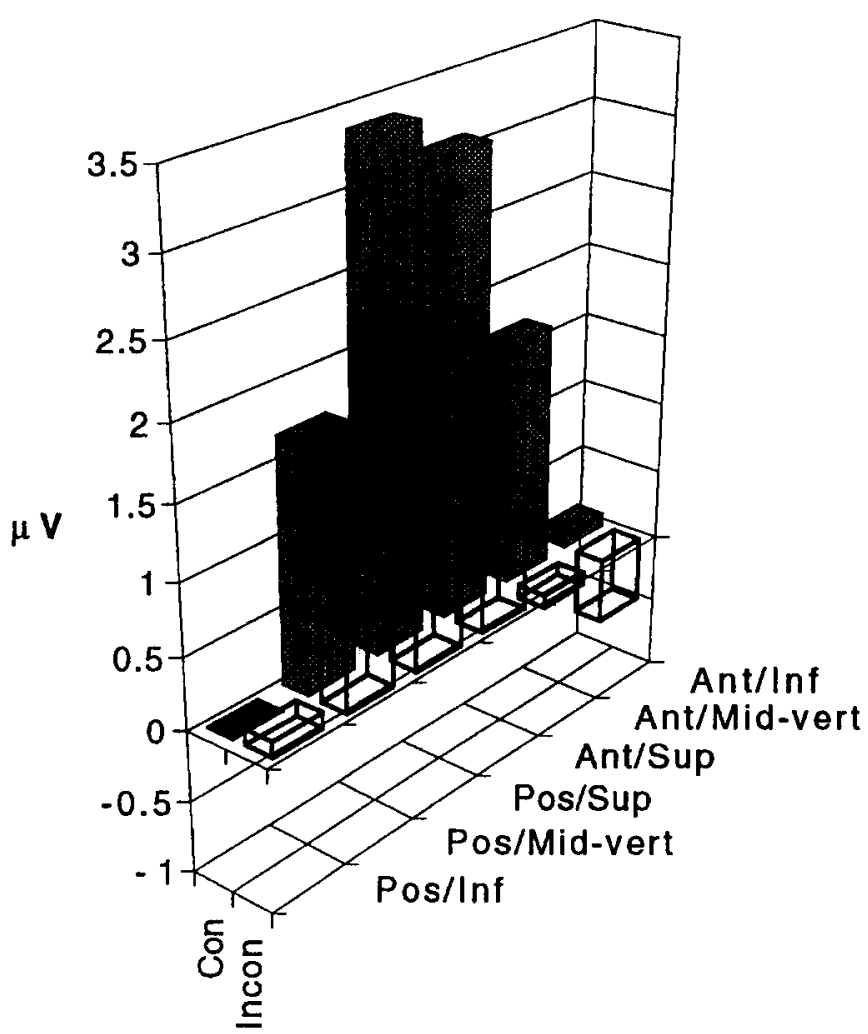

\section{Right Hemisphere}

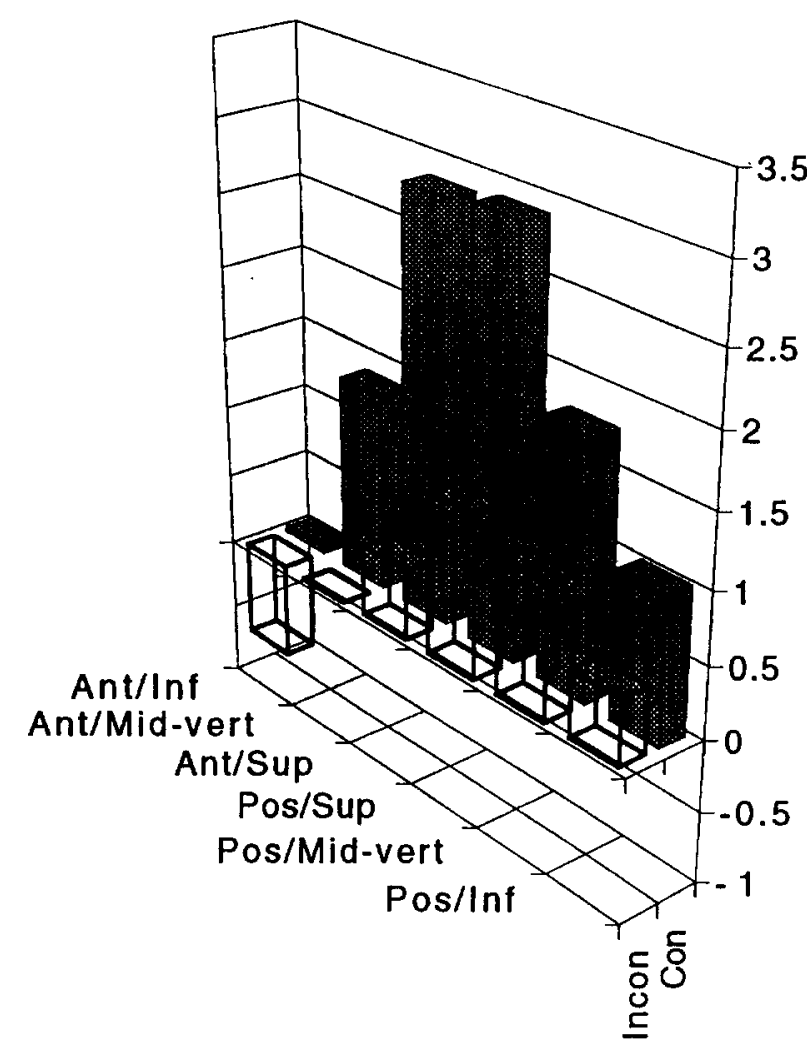

Fig. 11. Bar-chart representing the mean mastoid-referenced voltage for each of the 12 caudal $\times$ lateral $\times$ vertical cells in the intermediate-latency $(277-527 \mathrm{msec})$ ANOVA.

factor, indicating that the congruity effect was greater at anterior than posterior sites and at superior than inferior sites. These interactions are tempered by higher order interactions involving the sentence type and the spatial factors. There was a significant 3-way interaction involving sentence type, the lateral distribution, and the vertical distribution, $F(2,42)=8.53, \mathrm{MSe}=$ $0.23, P=0.0015$ as well as a 4-way interaction between the previous 3 factors and the caudal distribution, $F(2$, $42)=3.92$, $\mathrm{MSe}=0.25, P=0.03$. As demonstrated in Fig. 11 (also see Fig. 2), these interactions indicate that the mastoid-referenced congruity effect is greatest at the superior-frontal region of the left hemisphere.
Previous reports by Kutas (Kutas and Hillyard 1982; Kutas and Van Petten 1988) have shown that familial handedness often interacts with the laterality of the congruity effect. However, the sentence type by familial handedness by laterality interaction was only marginally significant in the present data, $F(1,21)=$ $3.52, \mathrm{MSe}=0.72, P=0.0747$. This trend suggests a greater congruity effect over the right hemisphere for subjects with left-handers in the family. No other laterality effects involving familial handedness reached significance.

A number of significant effects involving the spatial factors were obtained. The posterior voltages were

Fig. 8, $408 \mathrm{msec}$. Initial onset of the LPC for the incongruous word. This also appears to begin with what might be described as another P1 reprise, although it is somewhat more diffuse and superior than the earlier ones and it is right-sided rather than bilateral. The LPC for the congruous word has developed focal sources in the temporal area in the rCD data.

Fig. 9, 464 msec. Development of the LPC for the incongruous word. At this point, the congruous word ERP shows particularly strong source/sink gradients over the left temporal area.

Fig. 10, 584 msec. Peak of the LPC for the incongruous word. In addition to the anterior inferior negativities in the rV data, the rCD images show that for the incongruous word there is a particularly strong focal current source over the temporal area for the right hemisphere. 
significantly more positive than the anterior voltages. The inferior channels were more positive than the superior channels. The caudal factor interacted with the vertical factor due to a greater anterior/posterior differentiation in the inferior than in the superior channels. Finally, the lateral and vertical distributions interacted such that the inferior right hemisphere was more positive than the inferior left, but the reverse was true for the superior regions of the two hemispheres.

\section{$r V$ data}

P1 latency window $(64-120 \mathrm{msec})$. No effects involving the sentence type factor were reliable. Familial handedness interacted with the lateral distribution, $F$ $(1,21)=5.25, \mathrm{MSe}=0.86, P=0.03$, such that the $\mathrm{rV}$ of familial right handers was more positive over the left hemisphere, but the reverse was true for familial left handers. The highest-order spatial interaction to reach significance was between the caudality and verticality factors, $F(2,42)=7.36, \mathrm{MSe}=0.28, P=0.003$. This interaction confirms that the current flow at this time is more positive posteriorly and negative anteriorly. The posterior positivity of the P1 is greatest in the mid-vertical plane, as can be seen in Figs. 3 and 4.

\section{Left Hemisphere}

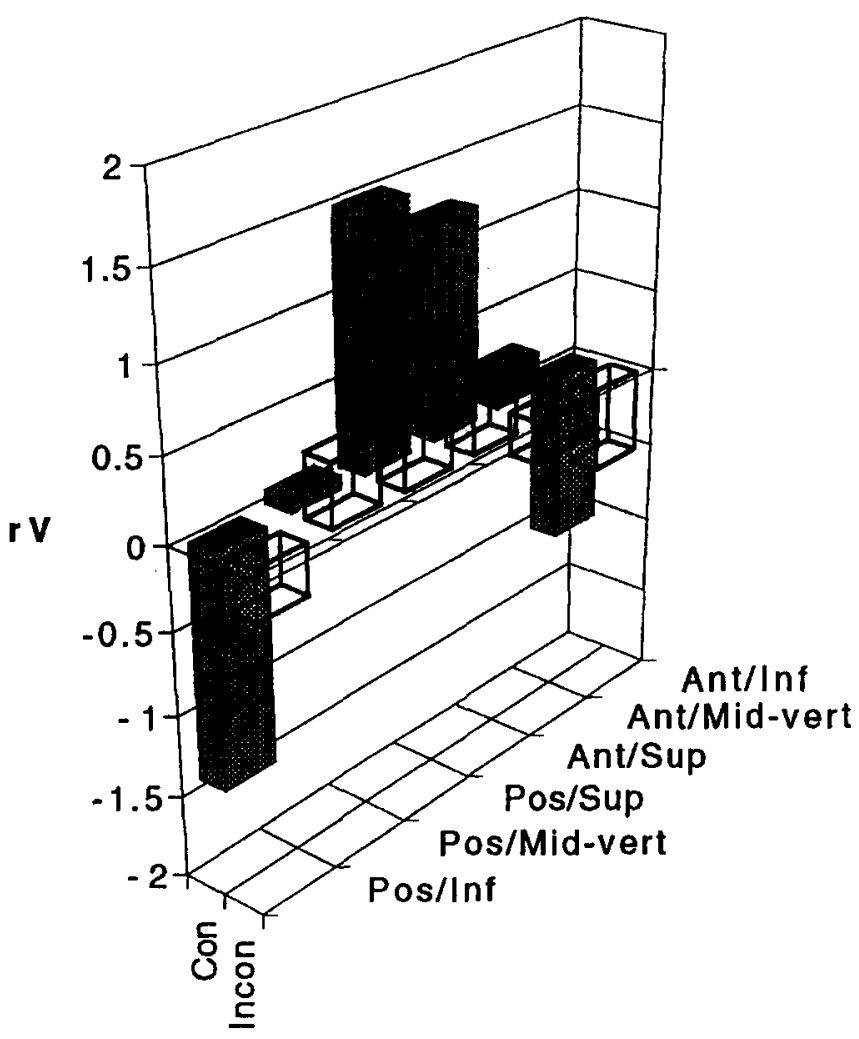

N1 latency window (136-184 msec). Congruous sentences were significantly more positive than incongruous sentences, $F(1,21)=5.73$, MSe $=0.06, P=$ 0.03. The lack of interactions between sentence type and the spatial factors indicates that this congruity effect is similar across the scalp. There were a number of significant interactions between the 3 spatial factors, culminating in a caudal by lateral by vertical interaction, $F(2,42)=16.90, \mathrm{MSe}=0.19, P=0.0001$. This interaction confirms that the $\mathrm{N} 1$ is maximally negative at the left posterior inferior area (Figs. 3 and 5).

$P 2$ latency window $(184-248 \mathrm{msec}$ ). The sentence type by vertical distribution interaction approached significance, $F(2,42)=3.08, \mathrm{MSe}=0.33, P=0.075$. This trend suggests that the congruous words are slightly more positive than incongruous at superior sites while the congruous are more negative at inferior sites. This effect is qualitatively similar to the strong intermediate latency congruity effect described below, so it is likely that this analysis has detected the onset of the intermediate latency, i.e., classical N400, effect.

The significant interaction between the 3 spatial factors, $F(2,42)=10.79$, $\mathrm{MSe}=0.19, P=0.0002$, reveals that the $\mathrm{rV}$ gradient between the vertex positivity

\section{Right Hemisphere}

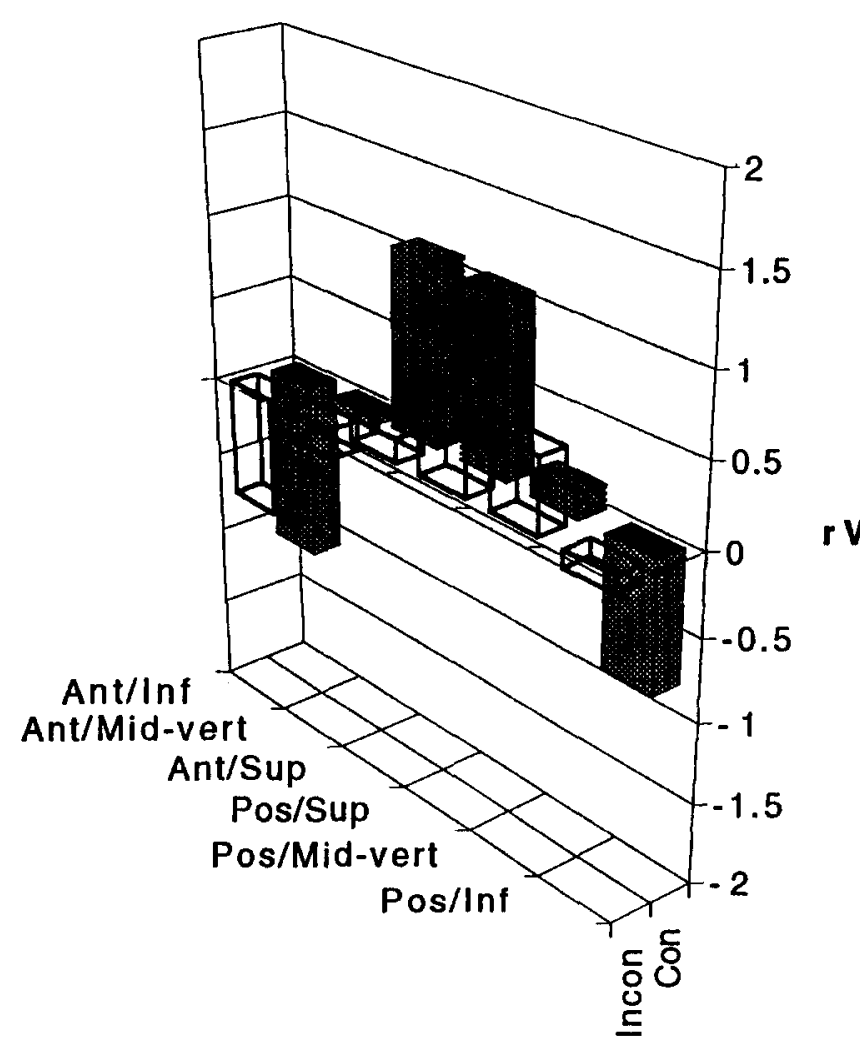

Fig. 12. Bar-chart representing the mean average potential gradient estimates (rV) for each of the 12 caudal $\times$ lateral $\times$ vertical cells in the intermediate-latency (277-527 msec) ANOVA. 
of the P2 and the posterior, inferior negativity associated with the $\mathrm{N} 1$ is greatest on the left side (Fig. 5).

Intermediate latency window (280-304 msec). There was not a significant main effect for sentence type in this analysis of the $\mathrm{rV}$ data $(F(1,21)=2.381$, MSe $=$ $0.16, P=0.14$ ). However, the effects of congruity became clear when the interactions between sentence type and the spatial distribution factors were considered. Sentence type interacted with both the lateral ( $F$ $(1,21)=17.589, \mathrm{MSe}=0.47, P=0.0004)$ and vertical $(F(2,42)=25.41, \mathrm{MSe}=0.79, P=0.0001)$ distributions of $\mathrm{rV}$. The congruous sentences are more positive than the incongruous ones anteriorly, but more negative posteriorly. Examination of Fig. 12 reveals that this differentiation is primarily in the incongruous condition, whereas the congruous gradients remain caudally symmetric. In the vertical interaction the polarity of the congruity effect reverses from inferior to superior areas such that congruous is more positive than incongruous superiorly but incongruous is more positive inferiorly. In contrast with the caudality interaction, which showed only the incongruous sentences shifting polarity, the vertical interaction is primarily due a

\section{Left Hemisphere}

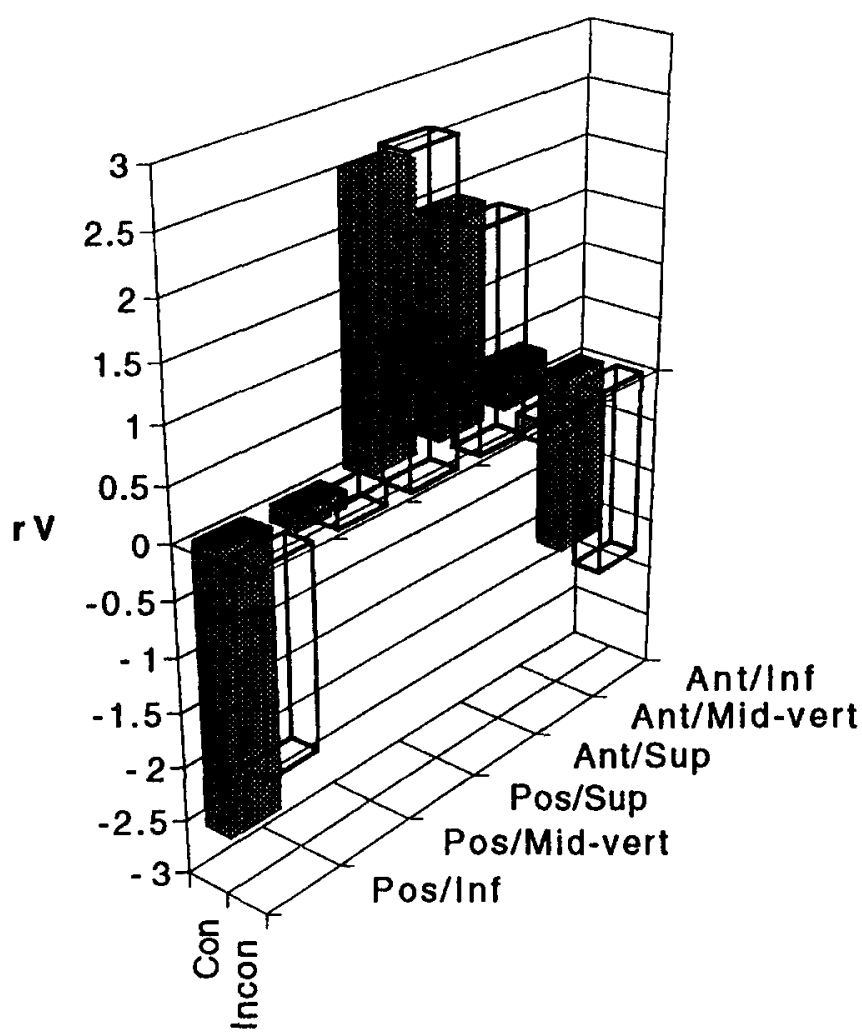

vertical polarity shift in the congruous sentences. An interaction between sentence type, vertical distribution, and the lateral distribution indicates that the vertical polarity shift in the congruity effect is maximal over the left hemisphere, $F(2,42)=5.99$, MSe $=0.18$, $P=0.008$. The 4-way interaction between the 3 spatial factors and sentence type was also significant, $F(2$, $42)=3.481, \mathrm{MSe}=0.21, P=0.04$. As seen in Fig. 12 (also Fig. 9), this interaction suggests that the congruity effect is strongest over the left posterior regions. The anterior congruity effect is attenuated primarily because the incongruous sentences are more negative anteriorly. The left hemisphere congruity effect is greater because the congruous amplitudes - both the inferior negativities and the superior positivities - are greater over the left than the right hemisphere.

A number of purely spatial interactions and main effects were significant. These can best be interpreted in the light of the interaction between the caudal and vertical distributions, $F(2,42)=3.42, \mathrm{MSe}=0.45, P$ $=0.04$. Inferior $\mathrm{rV}$ is consistently negative whereas the superior $\mathrm{rV}$ tends to be positive. This distinction is more apparent for the posterior than anterior fields.
Right Hemisphere

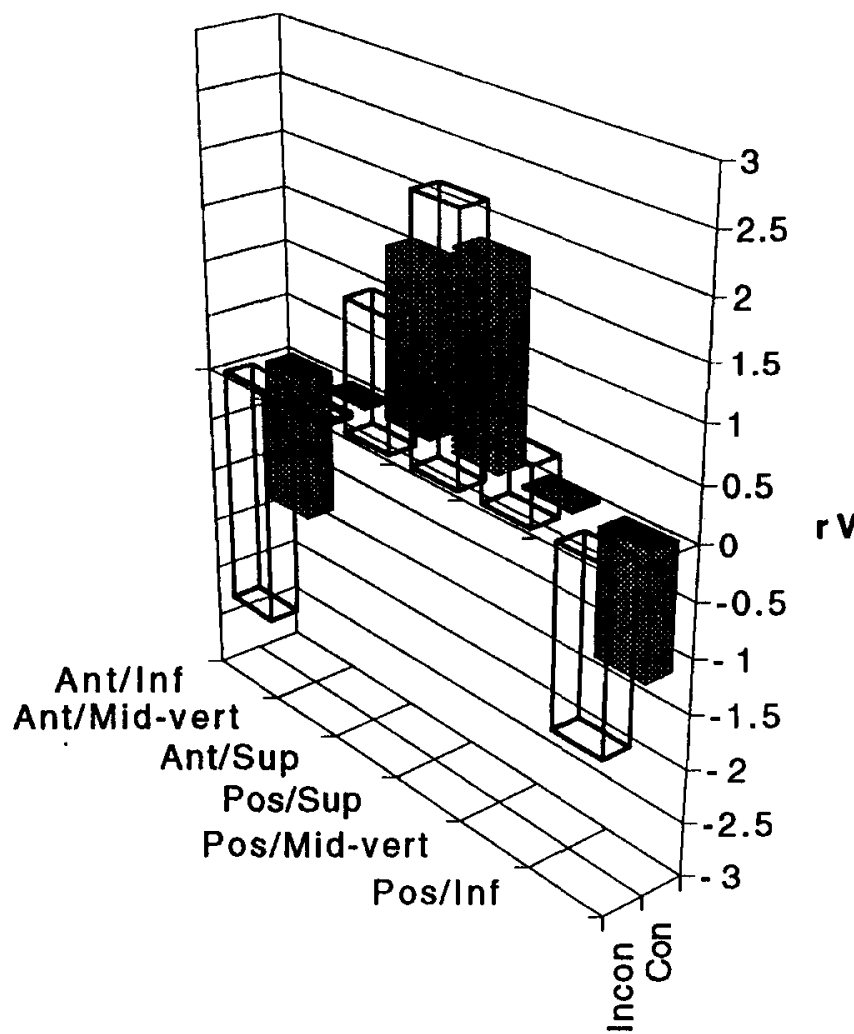

Fig. 13. Bar-chart representing the mean average potential gradient estimates $(\mathrm{rV})$ for each of the 12 caudal $\times$ lateral $\times$ vertical cells in the intermediate-latency (528-816 msec) ANOVA. 
Late window $(544-840 \mathrm{msec}$ ). Analysis of the late latency range revealed two distinct spatial interactions with the type of sentence ending. First, sentence type interacted with the caudal distribution of the $\mathrm{rV}, F(1$, $21)=13.97, \mathrm{MSe}=1.40, P=0.001$. This interaction is almost identical to the complimentary interaction involving the N4. As in the N4, congruous sentences are more positive than incongruous sentences anteriorly, but the polarity of this congruity effect reverses posteriorly. This interaction is primarily due to a shift in the incongruous $\mathrm{rV}$ data from anterior negativity to posterior positivity. In contrast, the distribution of the congruous $\mathrm{rV}$ is stable from anterior to posterior. The 3-way interaction between sentence type, lateral distribution and vertical distribution was also reliable, $F(2$, 42 ) $=9.14 \mathrm{MSe}=0.65, P=0.0006$. This interaction (see Figs. 13 and 10) suggests that the congruity effect for the late window becomes maximal over the right hemisphere, especially at the inferior locations.

There was a significant main effect involving the vertical distribution of the $\mathrm{rV}$, as well as a reliable vertical by lateral interaction. This interaction reveals that superior regions are more positive than the inferior regions. This main effect is greater in the left hemisphere compared to the right.

\section{Sentence recognition data}

After each block of sentences, the subject's memory for a subset of the sentences presented in that block was tested. The subject was shown a list of sentences with the final word missing. Some of the sentences were presented in the last block (half ended congruously, half incongruously) and others were new. For each subject, hit rates (probability of calling a previously presented sentence "old") were computed separately for the congruous and incongruous sentences. A single false alarm (FA) rate (probability of calling a never-before-seen sentence "old") was computed for each subject for the distractor sentences. These hits and false alarms were used to compute a bias-independent measure of recognition memory $\left(d^{\prime}=z_{\mathrm{FA}}-\right.$ $z_{\text {Hit }}$ ). Subjects recognized the beginnings of sentences ending congruously $\left(d^{\prime}=2.906\right)$ significantly better than those ending incongruously $\left(d^{\prime}=2.325\right), F(1$, 22) $=42.599$, $\mathrm{MSe}=0.091, P=0.0001$.

An exploratory correlational analysis was conducted to determine if average recognition ability correlated with any of the electrophysiological measures obtained during the presentation of the final word of the sentences. Pearson product moment correlations were computed between each subject's $d^{\prime}$ and the mean amplitude of the $\mathrm{rV}$ within the intermediate and late latency windows. Correlation coefficients were only computed between $d^{\prime}$ and mean amplitudes within each sentence type (i.e., ERPs from congruous words were only correlated with the congruous sentence $d^{\prime}$ 's).
These correlations were computed separately for each of the 12 cells defined by the 3 spatial factors used in the ANOVAs.

A number of significant correlations were obtained between $d^{\prime}$ and the $\mathrm{rV}$ from the intermediate latency region. Because a total of 146 correlations were computed within each of the latency windows, many chance effects would be expected if the measures were independent. However, the direction of the correlations appeared to be consistent with the ERP effects rather than random. The following spatial areas yielded $\mathrm{rV}$ values that significantly correlated with congruous $d^{\prime}$ : left, anterior, superior $(r=0.48, P=0.02)$; left, posterior, inferior $(r=-0.446, P=0.03)$; left, posterior, superior $(r=0.48, P=0.02)$; right, anterior, superior ( $r=0.44, P=0.036$ ); and right, posterior, inferior ( $r=$ $-0.60, P=0.002)$. For the incongruous sentences only the right, posterior, inferior $\mathrm{rV}$ correlated with $d^{\prime}$ ( $r=-0.42, P=0.04$ ). Within the late latency window, congruous $d^{\prime}$ was negatively correlated with the amplitude of $\mathrm{rV}$ in the right, posterior, inferior area $(r=$ $-0.42, P=0.05$ ).

Thus a consistent pattern emerged in which the negative correlations were associated with the inferior regions whereas the positive correlations were associated with the superior regions. This pattern indicates that good recognition was associated with a larger LPC. This effect appeared to be a general one for the congruous word, whereas for the incongruous word only the right posterior LPC showed a relation to correct recognition.

\section{Discussion}

Examining the conventional mastoid-referenced data, the present results replicate the previous findings of greater negativity in the ERP at about $400 \mathrm{msec}$ for words that are semantically incongruous with the sentence context (e.g., Kutas and Hillyard 1980b; Kutas and Van Petten 1988). Having observed this effect, the goal of our research was to examine its scalp topography. The two primary methodological improvements in the present study were the increased spatial resolution afforded by the 64-channel electrode array and the reference-independent characterization of brain electrical fields. By animating the average gradient (rV) and radial current density ( $\mathrm{rCD}$ ) data sample-by-sample, it was possible to visualize the temporal as well as spatial patterning in the data. These animations provided several interesting perspectives on the visual ERP during word reading. Certain features of the ERP such as the P1 and N1 were found to have highly focal scalp distributions. Interestingly, based on scalp topography, these data appeared to contain two P1 events, one at the typical P1 latency and another later in the 
epoch just prior to the onset of the LPC. Finally, the most sensitive indicator of the cognitive processing of the stimulus words, the N400, was found to be distinguished by an interval which was remarkable for the absence rather than the presence of topographic features.

\section{Identification of surface electrical events}

The first task in analyzing brain electrical activity recorded at the scalp surface is differentiating the features of the complex, dynamic patterns of head electrical fields. In early ERP work, this was accomplished by examination of the morphology of the wave form of a single channel, describing "components" either in terms of the sequence of the peaks and valleys (e.g., N1) or their latencies (e.g., N170). Neither of these conventions is adequate when dealing with data from a dense array. For example, the first negative deflection or "N1" in frontal channels in the present visual ERP can be seen to result from the diffuse, weak, negative polarity of the strong focal P1s in the back of the head (see the rV data mapped in Fig. 4). When the head is examined in its entirety, and the electrical fields are mapped in their dynamic course over time, the obvious phenomenological features simply cannot be captured by a definitional scheme that focuses on deflections at single channels. In this example of from the P1 interval, the conventional nomenclature is inadequate not only for distinguishing the sources of channel wave form bumps but for describing their polarity as well. This is also the case for the $\mathrm{P} 300$ or LPC in the present data. This event is a late "positive" complex only by virtue of the historical convention of using an inferior site such as ear or nose as a reference electrode. In a description of the whole head electrical fields, the LPC is as accurately described by the inferior negative potentials (current sinks) as by the superior positive potentials (current sources).

\section{Focal characterization of $P 1$ and $N 1$}

For many subjects, the P1 and N1 were sharply localized in the 64-channel $\mathrm{rV}$ data, often to individual electrode sites. Although the geodesic electrode net was positioned with reference to skull landmarks, there are large individual differences in brain and skull anatomy. The grand average maps shown in Figs. 4-10 thus reflect the localization trends of group data rather than the differentiated electrical fields of an actual brain. The highly focal nature of the P1s and N1s in many subjects' ERPs suggests that greater channel density (e.g., 128) may be required for full characterization of these components. A similar conclusion has been made for somatosensory ERPs on both theoretical and empirical grounds (Spitzer et al. 1989; Gevins 1990). In the present data, it was apparent that for many subjects the focal features of the ERP would have been poorly characterized or missed entirely with a sparse electrode array.

The N1 in the present study was strongly lateralized to the left hemisphere, for both congruous and incongruous words. This finding is consistent with previous reports of visual word ERPs in which semantic processing of the word was required (e.g., Neville et al. 1986). In other word-reading ERP studies in our laboratory (e.g., Compton et al. 1991), the N1 has shown a fairly symmetric bilateral distribution when semantic demands are minimal (such as in attending to features of the letters or in judging whether the stimulus is a word or nonword). Further studies with dense electrode arrays are necessary to determine whether differing demands for language processing alter the topography of the $\mathrm{N} 1$ and associated early negativities.

\section{Reprise of the P1 topography}

The spherical spline animations of the $\mathrm{rV}$ data show that the topography of the $\mathrm{P} 1$ - comprised of bilateral occipitoparietal positive foci - reappeared after the $\mathrm{N} 1 / \mathrm{P} 2$ interval. Although it is possible that different neural generators could produce the $P 1$ and the reprise of its topography later in the ERP epoch, it seems unlikely. Given the peak of P1 at 104 msec post stimulus, it also seems unlikely that the $\mathrm{P} 1$ reprise, with a peak in the vicinity of $312 \mathrm{msec}$ (Fig. 6), was a response to the offset of the word stimulus, which occurred at $105 \mathrm{msec}$. However, an important test of this reasoning will be to determine whether the phenomenon remains if the word offset is delayed, for example for $1 \mathrm{sec}$.

Using a limited montage of electrodes, what we have called the $\mathrm{P} 1$ reprise might have been considered a $\mathrm{P} 2$, since it would have been the second positive peak in many of the posterior recordings. However, this feature was clearly different from the large central positivity that began just after the onset of the N1 (Fig. 5 ). When examining a voltage wave form, it may appear as if one component, such as the $\mathrm{P} 2$, replaces a previous component, such as $\mathrm{N} 1$. The $\mathrm{rV}$ maps show that the anterior $\mathrm{P} 2$ foci occupied quite different locations than the $\mathrm{N} 1$ foci, and that these events were in fact coincident for the majority of their duration rather than sequential.

After the N1/P2 pattern, the congruous word ERP showed a P1 reprise in the superior occipital region that immediately developed into the LPC. For these data, the P1 reprise could be attributed to the onset of the LPC. However, the ERP to the incongruous word also showed a P1 reprise (Fig. 6) that was then followed not by an LPC but by the electrically silent period of the $\mathrm{N} 400$ (Fig. 7). Thus the P1 reprises for the two words appeared to be closely synchronized in time, even though their LPCs were not. Furthermore, when the LPC did appear for the incongruous word, it 
was preceded by a parietal positive wave on the right side that suggested a second $P 1$ reprise (Fig. 8).

Although the LPC or P300 has often been described as widespread, without localization to the sensory modality of the eliciting stimulus (e.g., Polich et al. 1991), the P1 reprises in the present congruous condition appeared to mark the onset of the LPC with renewed activity in the visual areas. It will be important to apply dense array topographic analysis to determine whether the P300s in auditory and somatosensory ERPs are initiated by modality-specific reprises of P1s in those areas as well. Systematic manipulation of perceptual and cognitive variables may help clarify the relations between the $P 1$, the apparent reprise of its topography, and the LPC.

\section{Topography of the N400}

The manipulation of semantic congruity produced clear effects by around $350 \mathrm{msec}$, as the ERP to the congruous word developed an LPC while that for the incongruous word did not. The congruous $\mathrm{rV}$ values were positive (sources) at the vertex, but negative (sinks) at inferior locations; this vertical differentiation was most clear over the posterior left hemisphere. The data for the incongruous words did not begin to show this vertical source/sink differentiation until $100-200 \mathrm{msec}$ later.

This pattern of vertex positivity and inferior negativity is quite similar to the scalp topography of the P300 we have observed in 64-channel $\mathrm{rV}$ data in target detection tasks (Potts et al., in preparation). Using an average-reference derivation, Skrandies and Lehmann (Skrandies 1983; Lehmann and Skrandies 1985) observed a similar topography for the $\mathrm{P} 300$, although their 16-channel montage over the occipito-parietal area was unlikely to characterize the whole head potentials (Tomberg et al. 1990).

An important question in ERP research has been the extent to which the $\mathrm{N} 400$ response to semantic incongruity appears because of a delay of the LPC. If the extended processing of a word, caused by its incongruity with the sentence, leads to a delay in the LPC, the N400 effect could appear in direct proportion to that delay. Kutas and Hillyard (1980a,b) showed that a P300 to unexpectedly large words was unlike the N400 produced by semantic incongruity. However, Polich $(1985 \mathrm{a}, \mathrm{b})$ has shown that the size of the N400 semantic congruity effect varies with the amplitude and latency of a subsequent $\mathbf{P} 300$. Thus, it has been suggested that the N400 may be just a later manifestation of the N2 that is normally associated with physical incongruities (Polich 1985b; Herning et al. 1987). Recently, Deacon et al. (1991) showed that having subjects perform either a physical (size) discrimination or a semantic discrimination on word pairs yielded an N2 for the physical discrimination and an N400 for the semantic discrimi- nation. They concluded that the only difference between these components was their latency.

An important factor for both the $\mathrm{N} 2$ and the $\mathrm{N} 400$ features of the ERP wave form may be the latency of the subsequent LPC. In the present experiment, the semantic congruity effect was seen as the difference between the ERP to the congruous word, which developed a strong, early LPC, and that to the incongruous word, which showed an interval of minimal topographic differentiation prior to the development of a delayed LPC. However, the semantic effect was indexed not only by the timing of the LPC, but its regional topography as well. For the relatively early LPC to the congruous word, the vertical source/sink differentiation of the LPC was greater over the left hemisphere than the right, and this lateralization was maintained for several hundred milliseconds. As the LPC developed for the incongruous word, it was laterally symmetric.

Although the LPC has typically been assumed to be a positive wave, the focal inferior negativities we observed for this phenomenon in the $\mathrm{rV}$ data may be as important as localizing features as the more diffuse superior positivity. In the ANOVAs, the congruity effect was greatest over the left posterior region (Fig. 12). This localization may be consistent with the indications from MEG and intracranial studies of a left medial temporal lobe generator for the semantic congruity effect (McCarthy and Wood 1984; Kutas and Van Petten 1988; Schmidt et al. 1989).

In the rCD maps for the LPC interval, the general pattern of inferior current sinks seen in the rV data was punctuated by focal current sources (positivities). These strong current sources were observed not at the superior sites to which the LPC is typically ascribed in the literature, but over the more inferior sites of temporal and orbital frontal regions. Consistent with the lateralization trends for the $\mathrm{rV}$ data described above, the rCD sources over temporal areas were left-lateralized in the early LPC for the congruous words (Fig. 9), but strongly right-lateralized for the later developing LPC for the incongruous words (Fig. 10). This pattern of results may be relevant to the hypothesis that the left hemisphere is specialized for processing highly constrained semantic information, whereas the right hemisphere is more important to processing semantically ambiguous or novel information (Burgess and Simpson 1988; Beeman 1990; Nakagawa 1991).

\section{Possible clues to mechanisms}

If the topographies of the LPC and the N400 are relevant to cognitive processing, they should show systematic relations with performance measures. Previous research has shown that, for the final words of sentences in an N400 paradigm, the words with larger LPCs will later be better remembered (e.g., Neville et al. 1986). In the present research, we assessed recogni- 
tion memory for the sentences themselves, and found it to be related to both the congruity of the final word and the LPC to that word. Sentences that were followed by a congruous word were correctly recognized more often than those followed by an incongruous word. Furthermore, regardless of the congruity of the ending word, if the LPC to that word was large, the sentence that preceded it was better remembered (Kutas 1988; Besson et al. in press). These data provide an interesting twist on the usual inference about the direction of semantic constraint in N400 experiments. Previous studies have considered how the semantic context of the sentence influences the cognitive processing of the final (or target) word. In the present results, the memory for the sentence stem itself appeared to be influenced by the semantic resolution provided by the final word, even when that word was omitted from the memory test. Thus, not only does a semantic context constrain the encoding of new information; the congruity of the information constrains the ongoing consolidation of the previous semantic context.

Although this experiment was not designed to test hypotheses about memory mechanisms, the dynamic changes in ERP topography included several features that may be relevant to current questions of the anatomical basis of semantic memory. Although their functional significance is only beginning to be understood, the focal, modality-specific ERP events, the P1 and N1, appear to index some aspects of the information processing in the visual system during reading. In addition, particularly in the $\mathrm{rCD}$ derivations, there were focal electrical events over frontal regions that appeared to parallel the processes occurring in the posterior brain. Such frontal events may shed light on the question of how broadly distributed cortical networks coordinate their operations to achieve coherent cognitive representations. In PET studies, Posner et al. (1988) observed increased inferior left frontal blood flow when subjects were given instructions to make semantic (category membership) decisions as they read single words. Although conventional neuropsychological theory has assumed that processing in the cortex is sequential (Geschwind 1965), such that sensory integration of the visual word form would be completed before semantic associations are developed, recent network models of language representation allow for greater interaction between the semantic and feature levels. In layered network models of word recognition (Rumelhart and McClelland 1986) the connection weights at a higher, lexical level of representation may act to constrain the weights being formed at the elementary featural level. If the frontal events of the ERP are found to be reliable neural phenomena, they may shed light on the interaction of frontal lobe operations with the representations taking shape in the posterior brain.
In addition to the several frontal events, the rCD derivations showed that the sharpest gradients in the scalp LPC field, and thus the most focal current sources, were often over temporal areas. It seems quite likely that these foci were influenced by the distortion of current flow around the ear canals. However, these foci did not remain fixed in the $\mathrm{rCD}$ animations, as one might expect if they were caused solely by anatomical factors. Rather, they shifted in location repeatedly during the LPC interval. These foci may indicate particularly strong regions of neural activity in the temporal lobes during the LPC. If so, it is tempting to think that an improved analysis of brain electrical events recorded at the scalp may be able to illuminate the interactions between sensory and paralimbic cortex in the process of memory formation.

The participation of limbic structures is essential to memory formation (Squire 1987). However, because prior memories can be recalled after limbic damage, the storage itself must take place in neocortex, probably in modality-specific pathways. Understanding the interaction between neocortical and limbic networks during the process of memory formation is one of the key challenges for cognitive neuroscience. The organization of the cortex converges the sensory pathways on the paralimbic cortices with a highly regular connectional architecture (Pandya et al. 1988). Derryberry and Tucker (1991) and Tucker (1992) emphasize that the motivational and evaluative representations in paralimbic cortex may be integral to the selection of information for storage as memories become consolidated. They propose that a recursive form of processing is mediated by the regular projections from sensory areas toward paralimbic areas, and by the back-projections from paralimbic to neocortical networks (Pandya et al. 1988). Cognitive processing could thus be viewed as a kind of arbitration, through which new sensory information in neocortical areas combines with existing motivational and evaluative representations in paralimbic areas to determine the content of memories. The cyclic reappearance of the P1 pattern in the present data, and the development of the LPCs from this pattern, suggest that a clearer understanding of brain electrical activity may provide clues to recursive cortico-limbic interactions (Edelman 1989).

Although the initial purpose of our research was to examine the topography of the $\mathrm{N} 400$, the topographic analysis revealed a number of interesting electrical phenomena. For those that prove reliable, the analytic experimental methods of cognitive psychology provide the means for systematic research into their functional significance. However, it is already becoming apparent that the relation between cognitive operations and electrical events may not always be obvious. The primary index of semantic processing in ERP research, the $\mathrm{N} 400$, was characterized not by a unique scalp 
topography, but an interval in which the electrical terrain was featureless. The brain's neural networks were not necessarily inactive during this interval. They may have been quite active, asynchronously. Such results suggest that cognition may be seen not only in the light of brain electrical events, but also in the shadows.

We thank Paul Compton for hardware and software design and implementation; Mike Worden and Matt Rubin for interpolation code for the Geodesic Electrode Net coordinate system; Jonathan C. Hansen and Steven Hillyard at UCSD for the spherical spline code; Gil Osgood for experiment control and data acquisition software; Peter Grossenbacher and Ray Vukcevich for wave form plot graphics; Daren Jackson, Michael Murias, and Chad Botwell for Net construction; Chris Ferry and Paul May for help in data acquisition; and Mario Liotti and Geoff Potts for help in data analysis and interpretation.

\section{References}

Beeman, M. (1990) Coherence inferencing and structure building in the cerebral hemispheres. Unpublished doctoral thesis, University of Oregon.

Bertrand, O., Perin, F. and Pernier, J. (1985) A theoretical justification of the average reference in topographic evoked potential studies. Electroenceph. clin. Neurophysiol., 62, 462-464.

Bloom, P.A. and Fischler, I. (1980) Completion norms for 329 sentence contexts. Mem. Cognit., 8, 631-642.

Burgess, C. and Simpson, G.B. (1988) Cerebral hemispheric mechanisms in the retrieval of ambiguous word meanings. Brain Lang., 33, 86-103.

Compton, P., Grossenbacher, P., Posner, M.I. and Tucker, D.M. (1991) A cognitive-anatomical approach to attention in lexical access. J. Cogn. Neurosci., 3, 304-312.

Deacon, D., Breton, F., Ritter, W. and Vaughan, Jr., H.G. (1991) The relationship between N2 and N400: scalp distribution, stimulus probability and task relevance. Psychophysiology, 28, 185-200.

Derryberry, D. and Tucker, D.M. (1991) The adaptive base of the neural hierarchy: elementary motivational controls on network function. In: R. Dienstbier (Ed.), Nebraska Symposium on Motivation. Univ. of Nebraska Press, Lincoln, NE.

Edelman, G.M. (1989) The remembered present: a biological theory of consciousness. Basic Books, New York.

Gevins, A. (1990) Distribted neuroelectric patterns of human neocortex during simple cognitive tasks. In: H.B.M. Uylings, C.G. Van Eden, J.P.C. De Bruin, M.A. Corner and M.G.P. Feenstra (Eds.), Progress in Brain Research, Vol 85. Elsevier Science Publishers, Amsterdam.

Geschwind, N. (1965) Disconnection syndromes in animals and man. Brain, 88, 237-294.

Halgren, E. and Smith, M.E. (1987) Cognitive evoked potentials as modulatory processes in human memory formation and retrieval. Hum. Neurobiol., 6, 129-139.

Herning, R.I.,Speer, M. and Jones, R.T. (1987) Event-related potentials to spoken equations: is the N400 really a late N200? Electroenceph. clin. Neurophysiol., Suppl. 40, 394-398.

Hjorth, B. (1975) An on-line transformation of EEG scalp potentials into orthogonal source derivations. Electroenceph. clin. Neurophysiol., 39, 526-530.

Hjorth, B. (1980) Source derivation simplifies topographical EEG interpretation. Am. J. EEG Technol., 20, 121-132.

Kutas, M. and Hillyard, S.A. (1980a) Event-related potentials to semantically inappropriate and surprisingly large words. Biol. Psychol., 11, 99-116.

Kutas, M. and Hillyard, S.A. (1980b) Reading senseless sentences: brain potentials reflect semantic incongruity. Science, 207, 203205.

Kutas, M. and Hillyard, S.A. (1982) The lateral distribution of event-related potentials during sentence processing. Neuropsychologia, 20, 579-590.

Kutas, M. and Hillyard, S.A. (1984) Brain potentials during reading reflect word expectancy and semantic association. Nature, 307, $161-163$.

Kutas, M. and Van Petten, C. (1988) Event-related brain potential studies of language. In: P.K. Acles, J.R. Jennings and M.G.H Coles (Eds.), Advances in Psychophysiology. JAI Press, Greenwich, CT, pp. 139-187.

Kutas, M., Lindamood, T.E. and Hillyard, S.A. (1984) Word expectancy and event-related brain potentials during sentence processing. In: S. Kornblum and J. Renquin (Eds.), Preparatory States and Processes. Erlbaum Press, Englewood Cliffs, NJ, pp. 217-237.

Kutas, M., Hillyard, S.A. and Gazzaniga, M.S. (1988a) Processing of semantic anomaly by right and left hemispheres of commissurotomy patients. Evidence from event-related brain potentials. Brain, $111,553-576$.

Kutas, M., Van Petten, C. and Besson, M. (1988b) Event-related potential asymmetries during the reading of sentences. Electroenceph. clin. Neurophysiol., 69, 218-233.

Lehmann, D. and Skrandies, W. (1985) Spatial analysis of evoked potentials in man - a review. Prog. Neurobiol., 23, 227-250.

McCarthy, G. and Wood, C.C. (1984) Intracranial recorded event-related potentials during sentence processing. Soc. Neurosci. Abst., $10,847$.

Nakagawa, A. (1991) Role of the anterior and posterior attention networks in hemispheric asymmetries during lexical decisions. J. Cogn. Neurosci., 3, 313-321.

Neville, H.J., Kutas, M., Chesney, G. and Schmidt, A.L. (1986) Event-related potentials during the initial processing and recognition memory of congruous and incongruous words. J. Mem. Lang., 25, 75-92.

Nunez, P.L. (1981) Electrical Fields of the Brain. Oxford University Press, New York.

Nunez, P.L. (1990) Physical principles and neurophysiological mechanisms underlying event-related potentials. In: J.W. Rorhbach, R. Parasuraman and R. Johnson (Eds.), Event-Related Brain Potentials: Basic Issues and Applications. Oxford University Press, New York, pp. 19-36.

Offner, F.F. (1950) The EEG as potential mapping: the value of the average monopolar reference. Electroenceph. clin. Neurophysiol., $2,215-216$

Pandya, D.N., Seltzer, B. and Barbas, H. (1988) Input-output organization of the primate cerebral cortex. In: Comparative Primate Biology, Vol. IV: Neurosciences. Allen Ardlis, New York, pp. 39-80.

Perrin, F., Pernier, J., Bertrand, D. and Echallier, J.F. (1989) Spherical splines for scalp potential and current density mapping. Electroenceph. clin. Neurophysiol,, 72, 184-187. (Corrigendum: Electroenceph. clin. Neurophysiol., 1990, 76: 565.)

Polich, J. (1985a) N400s from sentences, semantic categories, number and letter strings? Bull. Psychon. Soc., 23, 361-364.

Polich, J. (1985b) Semantic categorization and event-related potentials. Brain Lang., 26, 304-321.

Polich, J. Brock, T. and Geisler, M.W. (1991) P300 from auditory and somatosensory stimuli: probability and inter-stimulus interval. Int. J. Psychophysiol., 11, 219-223.

Posner, M.I., Petersen, S.E., Fox, P.T. and Raichle, M.E. (1988) Localization of cognitive operations in the human brain. Science, $240,1627-1631$. 
Rumelhart, D.E. and McClelland, J.L. (1986) Parallel distributed processing: explorations in the microstructure of cognition. Vol I: Foundations. MIT Press, Cambridge, MA.

Schmidt, A.L., Arthur, D.L., Kutas, M. and Flynn, E. (1989) Neuromagnetic responses during reading meaningful and nonmeaningful sentences. Psychophysiol. Abst., 26, S6.

Skrandies, W. (1983) Information processing and evoked potentials: topography of early and late components. Adv. Biol. Psychiat., 13, $1-12$.

Smith, M.E., Stapleton, J.M. and Halgren, E. (1986) Human medial temporal lobe potentials evoked in memory and language tasks. Electroenceph. clin. Neurophysiol., 63, 145-159.

Spitzer, A.R., Cohen, L.G., Fabrikant, J. and Hallett, M. (1989) A method for determining optimal interelectrode spacing for cerebral topographic mapping. Electroenceph. clin. Neurophysiol., 72, 355-361.
Squire, L.R. (1987) Memory and Brain. Oxford University Press, New York.

Taylor, W.L. (1953) "Cloze" procedure: a new tool for measuring readability. Journalism Quart., 30, 415-417.

Tomberg, C., Noel, P., Ozaki, I. and Desmedt, J.E. (1990) Inadequacy of the average reference for the topographic mapping of focal enhancements of brain potentials. Electroenceph. clin. Neurophysiol., 77, 259-265.

Tucker, D.M. (1992) Development of emotion and cortical networks. In: M. Gunnar and C. Nelson (Eds.), Minnesota Symposium on Child Development: Developmental Neuroscience. Oxford Univ. Press, New York.

Van Petten, C. and Kutas, M. (1990) Interactions between sentence context and word frequency in event-related brain potentials. Mem. Cognit., 18, 380-393. 